\title{
Adequacy of Effective Born for electroweak effects and TauSpinner algorithms for high energy physics simulated samples
}

\author{
E. Richter-Was ${ }^{1} \mathbb{D}$, Z. $_{\text {. }}$ as $^{2, a}{ }_{\mathbb{D}}$ \\ ${ }^{1}$ Institute of Physics, Jagellonian University, ul. Lojasiewicza 11, 30-348 Kraków, Poland \\ 2 IFJ-PAN, ul. Radzikowskiego 152, 31-342 Kraków, Poland
}

Received: 13 February 2021 / Accepted: 16 December 2021

(C) The Author(s) 2022

\begin{abstract}
Matching and comparing the measurements of past and future experiments call for consistency checks of electroweak (EW) calculations used for their interpretation. On the other hand, new calculation schemes of the field theory can be beneficial for precision, even if they may obscure comparisons with earlier results. Over the years, concepts of Improved Born, Effective Born, as well as of effective couplings, in particular of $\sin ^{2} \theta_{W}^{e f f}$ mixing angle for EW interactions, have evolved. In our discussion, we use four versions of DIZET EW library for phenomenology of practically all HEP accelerator experiments over the last 30 years. We rely on the codes published and archived with the KKMC Monte Carlo program for $e^{+} e^{-} \rightarrow$ $f \bar{f} n(\gamma)$ and available for the Tauspinner as well. Tauspinner re-weighs generated events for introduction of EW effects. To this end, DIZET is first invoked, and its results are stored in data file and later used. Documentation of TauSpinner upgrade, to version 2.1.0, and that of its new arrangement for semi-automated benchmark plots are provided. In our paper, focus is placed on the numerical results, on the different approximations introduced in Improved Born to obtain Effective Born, which is simpler for applications of strong or QED processes in $p p$ or $e^{+} e^{-}$colliders. The $\tau$ lepton polarization $P_{\tau}$, forward-backward asymmetry $A_{F B}$ and parton-level total cross section $\sigma^{\text {tot }}$ are used to monitor the size of EW effects and effective $\sin ^{2} \theta_{W}^{e f f}$ picture limitations for precision physics. Collected results include: (i) Effective Born approximations and $\sin ^{2} \theta_{W}^{\text {eff }}$, (ii) differences between versions of EW libraries and (iii) parametric uncertainties due to, for example, $m_{t}$ or $\Delta \alpha_{h}^{(5)}(s)$. These results can be considered as benchmarks and also allow to evaluate the adequacy of Effective Born with respect to Improved Born. Definitions are addressed too.
\end{abstract}

\section{Introduction}

One of the great achievements of precision high energy physics in the electroweak (EW) sector measurements was establishing that quantum field theory can be indeed used for predictions that match measurements $[1,2]$. To handle results and interpretations, the concept of pseudo-observables (usually observables in idealized acceptance regions, which at least in principle can be measured) was very useful [3]. Over many years, the effective EW mixing

\footnotetext{
a e-mail: Z.Was@cern.ch (corresponding author)
} 
angle $\sin ^{2} \theta_{W}^{\text {eff } f}$ (of the process $e^{+} e^{-} \rightarrow Z / \gamma^{*} \rightarrow f \bar{f}$, where $f$ denote leptons or quarks) was a prime candidate to evaluate sensitivity of observations of the EW sectors and in fact established itself as universal pseudo-observable [2] too. This quantity represents ratio of vector and axial couplings of fermions to the $Z$ boson. To remain useful, it is needed that the approximations still hold at the required precision.

The $\sin ^{2} \theta_{W}^{e f f} f$ uncertainty of all measurements of SLD+LEP experiments combined, reached $16 \cdot 10^{-5}$ [2]. Only for FCC-ee, which is planned in relatively distant future, uncertainty is expected to be sizably reduced, to $0.6 \cdot 10^{-5}$ [4].

For LHC it reads: (i) ATLAS: $0.23140 \pm 21 \cdot 10^{-5}$ (stat) $\pm 24 \cdot 10^{-5}$ (PDF) $\pm 16 \cdot 10^{-5}$ (syst) in total $\pm 36 \cdot 10^{-5}$ [5]. (ii) CMS: $0.23101 \pm 36 \cdot 10^{-5}$ (stat) $\pm 18 \cdot 10^{-5}$ (syst) $\pm 16 \cdot 10^{-5}$ (theo) $\pm 31 \cdot 10^{-5}$ (PDF) total $\pm 53 \cdot 10^{-5}$ [6]. (iii) HL-LHC ATLAS prospects: $\pm 18 \cdot 10^{-5}$ (total) and $\pm 15 \cdot 10^{-5}$ (PDF) [7]. (iv) HL-LHC CMS prospects: with possible detector upgrade may help to reduce PDF uncertainty to $\pm 12 \cdot 10^{-5}$ [8].

One observes, that in all LHC cases, dominant uncertainties come from description of strong interactions. That includes uncertainties from parton distribution functions (PDF)s, configurations with additional jets and multi-loop corrections of QCD. That is where the bulk of effort is necessary. To ease the difficulties, it is convenient to eliminate or reduce complications due to electroweak effects in other parts of calculations. Note that for LHC measurements, systematic error of $35 \cdot 10^{-5}$ was achieved so far and is expected to reach $18 \cdot 10^{-5}$. Then, it will become comparable to the one at SLD + LEP. The EW sector theoretical predictions and programs of that time should remain adequate, if proper implementation into $p p$ collision Monte Carlos is prepared.

In the calculation scheme used in LEP 1 times, EW corrections calculated at one-loop level were improved with selected, dominant higher-order terms and embedded in the Improved Born Approximation [9], which was a cornerstone for LEP measurements [1]. Significant effort was devoted to precision and theoretical foundations, not only of electroweak effects alone, but also how to match them with other parts of interactions. That involves consequences for factorization of phenomena, like initial/final state bremsstrahlung in case of $e^{+} e^{-}$collisions [10] or jet/PDFs in case of the $p p$ or $p \bar{p}$ [11]. General reasoning relies on the fact that other parts of processes are at smaller energy scale than electroweak ones (under condition that QED part is localized and removed). We will not repeat that discussions, but devote our attention to practical considerations, that is, how to combine electroweak effects with the results of calculations or simulations of strong interactions of $p p$ collisions for Drell-Yan (DY) production processes in vicinity of $Z$ resonance. Note that strong interactions are the dominant source of systematic uncertainties for precision electroweak measurements. This essential, but not central to our aims, point will be covered in Appendices. In the following, we will concentrate on simplifications for electroweak components, which may be essential in merging with strong interaction calculations. We will evaluate resulting loss of precision for electroweak results.

The Effective Born means re-definition of coupling constants, but analytic structure of Born amplitudes remains intact and such re-definition usually does not break properties required for factorizing out strong interaction effects. That is why, it was used for interpretation of LEP 2 data and is relatively easy to use for calculations of LHC, HL-LHC and FCC-hh phenomenology as well, where as we have pointed out, electroweak effects are not the main obstacle for precision. The main source of uncertainties are strong interactions.

In contrary, in an Improved Born (the most sophisticated picture developed at LEP 1 time) couplings are accompanied with energy- and angle-dependent, complex form factors. This can damage strong interaction gauge invariance and would imply necessity of simultaneous 
calculation for the EW and QCD effects of $p p$ collision processes. The energy and scattering angle dependence inevitably breaks Ward identities, see, for example, [12] or [13]. Complex coupling constants/form factors of electroweak sector are a concern for strong interaction calculations, where EW interactions are treated as external currents. See again, for example, [12]. That obstacle have been overcome for LEP 1 applications, because then, only $s$-channel EW boson exchange contributed. For the high precision channels, both initial and final state were leptonic. And QED initial, final and interference interactions including real photon bremsstrahlung were carefully studied and separated out. Thanks to that efforts, this part of interactions could have been treated separately and to higher orders, without the necessity to simultaneously addressing multi-loop effects in full second or even higher order of EW effects. Also strong interactions contributed at LEP 1 with vacuum polarization loops, thus corrections of photon and $Z$ boson propagators only. For LEP 2, precision requirements were not demanding, electroweak loop corrections and form factors were not needed, and Effective Born was enough.

The LEP 1 conditions of hard processes with energies essentially of the $Z$ peak do not need to hold for future applications. It is thus of interest what are the limitations of the previously used approaches, independently whether they are going to be sufficient, or for compatibility studies of previous results only.

Feasibility of effective coupling definitions relies on numerical properties of EW loop corrections. Comparisons with Improved Born could invalidate the whole concept of Effective Born and $\sin ^{2} \theta_{W}^{e f f}$ definition as well. In fact the concept of effective couplings has evolved over time [14-16]. The variants of the past, the controversies and differences in conventions can be now easily identified and confusions avoided. One should keep in mind that some conventions were used in data analyses. With the improving measurements precision, one has to readdress the validity of assumptions and approximations necessary for definitions and usefulness of Effective and Improved Born. Let us point, as an example, to an early reference [17], where dependence on flavour and the flavour-dependent $\sin ^{2} \theta_{W}^{e f f}$ was elaborated.

To address the above points, one needs to investigate first, if Improved or Effective Born can be localized and factorized with sufficient precision and kinematic details in calculations of strong interactions. References [18,19] were devoted to studying how the strong interaction separates in LHC processes of $W$ and $Z$ boson production and decay. The study was necessary for validation of TauSpinner event re-weighting algorithm [20,21] in its implementation of EW effects [22]. Even more sophisticated evaluations (see Sect. 2) were completed in the past, for $e^{+} e^{-}$collisions in context of Monte Carlo generators [10,23,24] and for semianalytical calculations in [9]. One of the important numerical assumptions was that in all these applications, numerical differences between Improved and Effective Born are, around the $Z$ pole, not too large. The one-loop genuine weak corrections were usually calculated for all these projects with the help of DIZET library [25]. Important was the inclusion of some higher-order strong interaction or QED effects. One has always to check if necessary higher-order contributions to weak loop effects can be (and are) introduced into Effective or Improved Born and thus partly re-summed as well. For DIZET archivization and evolution, see Ref. [26] and references therein.

Our attention is oriented toward numerical studies. Some conceptual points are nonetheless collected in Appendix A, which should be understood as encouragement for further reading. We also address the reader to recent Ref. [27] and references therein.

Whenever precision is expected to improve, assumptions behind calculations need to be revisited. The TauSpinner algorithms can be helpful in that respect and used to evaluate if for a given observable some classes of the corrections are necessary or can be ignored. 
Independently if old calculations are sufficient or if the new one may be necessary, it is useful to establish which of the effects need to be taken into account. The compatibility with monumental work encapsulated in DIZET / ZFITTER project is important also because most of LEP/Tevatron/LHC electroweak precision data analysis used these tools.

We focus on discussion/evaluation of: (i) the suitability of Effective versus Improved Born approximation (also the usefulness of $\sin ^{2} \theta_{W}^{e f f}$ ), (ii) differences between results of DIZET library versions in use over the last 30 years, (iii) ambiguities due to parametric uncertainties or due to (sometimes) missing contributions. We also explain TauSpinner extensions with respect to Ref. [22] that are minor, but useful for studies of EW effects.

In the scope of the paper, we present numerical results, either from semi-analytical calculations (predominantly for $e^{+} e^{-} \rightarrow l^{+} l^{-}$processes) or from re-weighting of the Monte Carlo event samples (predominantly for $p p \rightarrow Z / \gamma^{*} \rightarrow l^{+} l^{-}$processes at $8 \mathrm{TeV}$ centreof-mass collisions). In the second case, we use Powheg Monte Carlo $Z+$ jets events as described in Refs. [18,22].

Section 2 recalls concept of Improved Born and necessary for its introduction definitions, practical comments on $\sin ^{2} \theta_{W}^{e f f}$ definitions are provided. Simplifications enabling introduction of Effective Born, easier to be combined with other parts of calculations, are explained in the section as well. Details of the definition are given in Sect. 3.1. The main purpose of Sect. 3 is to recall numerical results on non-EW effects which are instrumental for the concept of Effective Born and effective couplings, first. Both Improved Born and Effective Born require interpolation of the $2 \rightarrow 2$ kinematics and evaluation of its scattering angle. This requires careful optimization in the presence of QED/QCD initial and final state emissions; see references above, which discuss the issue. In this context, re-definitions of couplings are convenient as such couplings can be usually used in strong interactions amplitudes without complication of gauge independence restoration. Alternatively, re-weighting to Improved Born can be advocated following the TauSpinner solution of Ref. [22]. The particular choice will need to be decided by the user precision requirements. That is why we do not give any guidelines here. Definitions of simplified test observables used all over the paper are provided.

Section 4 is devoted to the comparison of Improved Born and Effective Born. In particular, results useful to evaluate precision of Effective Born and $\sin ^{2} \theta_{W}^{e f f}$ approximations with respect to Improved Born are provided. Most of the numerical details are delegated to Appendices. Reference. [26] summarizes numerically most important upgrades of EW library DIZET. Some numerical comparisons of its variants are collected in Sect. 5. Numerical results for parametric uncertainties are given in Sect. 5.1. Summary, Sect. 6, closes the paper.

Some introduction, review and explanations of conceptual nature can be found in Appendix A. Technical and physics content details of DIZET library versions, including initialization parameters used by us in these cases, are collected in Appendix B. Alternative EW projects and calculation schemes are not discussed, and we address interested readers to the documentation of KKMC [24] or even older KORALZ documentation [23]. Appendix C supplements the paper with technical details on the TauSpinner re-weighting algorithm. Details of variants of Born definitions and activating them flags available in TauSpinner are given in Appendix D. Appendix E addresses the important but auxiliary point of the $Z$ propagator with running or fixed width and provides corresponding numerical results. Appendix F enumerates versions of DIZET library, which are available from Ref. [26]. 


\section{Improved Born and electroweak form factors.}

In the Improved Born Approximation, the complete $O(\alpha)$ EW corrections, supplemented by selected higher-order terms, are handled with form-factor corrections, dependent on ( $s$, $t$ ), multiplying couplings and propagators of the usual Born expressions. That is appropriate if Improved Born is treated independently from other parts of complete scattering process calculations. Otherwise an effort to justify why such result can be used as part of complete prediction is necessary. In some cases, it can be done, in others not, or may lead to substantial complications, or restricted applicability domain. See more discussion in Appendix A.

Let us continue with definition of Improved Born used in TauSpinner. It is detailed in Ref. [22], but we will recall it with Eq. (1) for the process $e^{+} e^{-} \rightarrow f \bar{f}$. The formula can be used also in the case when initial and final state is interchanged. The $z$ component of the fermion isospin $T_{3}^{e, f}$, EW mixing angle $s_{W}^{2}=\sin ^{2} \theta_{W}, c_{W}^{2}=1-s_{W}^{2}$ and electric charge $q_{e, f}$ is used, as usual, for the coupling constant calculations. The Mandelstam variables $s=\left(p_{e^{+}}+p_{e^{-}}\right)^{2}$ and $t=\left(p_{f}-p_{e^{-}}\right)^{2}$ are used for the kinematical dependence. The Fermi constant $G_{\mu}$, QED coupling constant $\alpha$, the $Z$ boson mass $M_{Z}$ and width $\Gamma_{Z}$ complete basic notations. Definitions of EW form factors $K_{e}(s, t), K_{f}(s, t), K_{e f}(s, t)$, for $\ell=e, \mu, \tau$, photon vacuum polarization $\Pi_{\gamma \gamma}(s)$ and $\rho_{\ell f}(s, t)$, are as used in [22]. It is important that they are only weakly dependent on $t$, and the $s$ dependence is not sizable as well, ${ }^{1}$ see Fig. 1.

$$
\begin{aligned}
& M E_{B o r n+E W}=\mathcal{N} \frac{\alpha}{s}\left\{\left[\bar{u} \gamma^{\mu} v g_{\mu \nu} \bar{v} \gamma^{\nu} u\right] \cdot\left(q_{e} \cdot q_{f}\right) \cdot \Gamma_{V_{\Pi}} \cdot \chi_{\gamma}(s)\right. \\
& +\left[\bar{u} \gamma^{\mu} v g_{\mu \nu} \bar{v} \gamma^{\nu} u \cdot\left(v_{e} \cdot v_{f} \cdot v v_{e f}\right)\right. \\
& +\bar{u} \gamma^{\mu} v g_{\mu \nu} \bar{v} \gamma^{v} \gamma^{5} u \cdot\left(v_{e} \cdot a_{f}\right) \\
& +\bar{u} \gamma^{\mu} \gamma^{5} v g_{\mu \nu} \bar{v} \gamma^{\nu} u \cdot\left(a_{e} \cdot v_{f}\right) \\
& \left.\left.+\bar{u} \gamma^{\mu} \gamma^{5} v g_{\mu \nu} \bar{v} \gamma^{v} \gamma^{5} u \cdot\left(a_{e} \cdot a_{f}\right)\right] \cdot Z_{V_{\Pi}} \cdot \chi_{Z}(s)\right\}, \\
& v_{e}=\left(2 \cdot T_{3}^{e}-4 \cdot q_{e} \cdot s_{W}^{2} \cdot K_{e}(s, t)\right) / \Delta \text {, } \\
& v_{f}=\left(2 \cdot T_{3}^{f}-4 \cdot q_{f} \cdot s_{W}^{2} \cdot K_{f}(s, t)\right) / \Delta \text {, } \\
& a_{e}=\left(2 \cdot T_{3}^{e}\right) / \Delta, \quad s_{W}^{2}=\left(1-c_{W}^{2}\right)=1-M_{W}^{2} / M_{Z}^{2}, \\
& a_{f}=\left(2 \cdot T_{3}^{f}\right) / \Delta, \quad \Delta=4 s_{W} c_{W}, \\
& \chi_{Z}(s)=\frac{G_{\mu} \cdot M_{z}^{2} \cdot \Delta^{2}}{\sqrt{2} \cdot 8 \pi \cdot \alpha} \cdot \frac{s}{s-M_{Z}^{2}+i \cdot \Gamma_{Z} \cdot s / M_{Z}}, \\
& \Gamma_{V_{\Pi}}=\frac{1}{2-\left(1+\Pi_{\gamma \gamma}(s)\right)}, \quad Z_{V_{\Pi}}=\rho_{\ell f}(s, t), \quad \chi_{\gamma}(s)=1, \\
& v v_{e f}=\frac{1}{v_{e} \cdot v_{f}}\left[\left(2 \cdot T_{3}^{e}\right)\left(2 \cdot T_{3}^{f}\right)-4 \cdot q_{e} \cdot s_{W}^{2} \cdot K_{f}(s, t)\right. \\
& -4 \cdot q_{f} \cdot s_{W}^{2} \cdot K_{e}(s, t)+\left(4 \cdot q_{e} \cdot s_{W}^{2}\right) \\
& \left.\left(4 \cdot q_{f} \cdot s_{W}^{2}\right) K_{e f}(s, t)\right] \frac{1}{\Delta^{2}} \text {. }
\end{aligned}
$$

\footnotetext{
${ }_{1}$ At the LO EW $K_{e}(s, t)=K_{f}(s, t)=\rho_{\ell f}(s, t)=1, \quad \Pi_{\gamma \gamma}(s)=0$ and $\frac{G_{\mu} M_{Z}^{2} \Delta^{2}}{\sqrt{2} \cdot 8 \pi \alpha}=1$. We use $s_{W}^{2}=$ $1-M_{W}^{2} / M_{Z}^{2}$ for the on-mass-shell definition. The $v v_{e f}=1$ at LO too. The $v v_{e f}-1$ bring correction which cannot be attributed to coupling or to propagators, but depends on the whole process.
} 
In the formula, $u, v$ stand for spinors-fermions wave functions, and $\mathcal{N}$ is a normalization factor which is convention dependent (e.g. for wave functions normalization). The $\left(G_{\mu}, M_{Z}\right.$, $\alpha(0))$ input scheme is used; for details, see Appendix B. Note that input includes also $\Pi_{\gamma \gamma}(s)$, $t_{t}, m_{h}$ and that $M_{W}$ is calculated from Eq. (6).

The formula (1) is not the only possibility for implementation of EW corrections. Genuine EW corrections can be, under some conditions, combined with the ones of QED or strong interactions. For example in KKMC implementation, the Improved or Effective Born approximation is not used. EW form factors are installed into spin amplitudes directly, but this required substantial effort, see Appendix A. In Appendix, separation of electroweak effects into parts which are easier to handle individually is described. The gauge invariance is helpful to identify initial state-, final state-, interference-box-corrections as well as vacuum polarization ones, for example, care of the QED gauge cancellation in spin amplitudes for $e^{+} e^{-} \rightarrow \operatorname{ll} n \gamma$. The "running" of $\Gamma_{Z}$ for the $\chi_{Z}(s)$ propagator is used in Eq. (1) as was commonly the case for LEP physics, but is less so for LHC oriented Monte Carlos (MC's). Another possibility is to use Effective Born. (Not exact at one-loop level, it will be discussed in Sect. 3.1.) The main idea is to simplify formula (1). In particular, include in $\sin ^{2} \theta_{W}^{e f f}$ the bulk effect of $K_{e}(s, t)$ and $K_{f}(s, t)$ form factors present in front of $s_{W}^{2}$, and vacuum polarization corrections $\Pi_{\gamma \gamma}$ into a re-definition of $\alpha$. In fact a real part or a module and both are calculated at the $Z$ pole. It is also possible to think of $\sin ^{2} \theta_{W}^{e f f}$ as the best result of the fit to the data. At certain precision level, such distinction may start to play a role and one should be aware of alternatives to avoid misunderstandings.

Definition of form factors follows the one of the DIZET libraries $[25,26]$ and is as used for spin amplitudes in the KKMC Monte Carlo as well. They are used in the Improved Born, formula (1), of Tauspinner too. Details of the EW scheme and initialization parameters are provided in Appendix B. In Fig. 1, EW form factors calculated with DIZET library are drawn.

A disadvantage of the Improved Born with respect to the Effective one is that it is not immediate to merge its formulation into calculations (programs) for strong interactions, e.g. for DY production in $p p$ collisions. Formula (1) can be used as a starting point to evaluate simplifications introduced in Improved Born to obtain simpler Effective Born, where $s$ - and $t$ dependent form factors are avoided. For the latter, form factors are often set to unity or may be replaced with the real constants, thus not compromising strong interaction calculations. One should keep in mind that numerical values for $s_{W}^{2}$ and $\alpha$ need then to be chosen differently, also to accumulate dominant numerical contributions of the loop corrections. Technical details for the arrangements used in the programs are collected in Table 13 of Appendix D.

The picture of Improved Born [9] remains a great achievement of LEP 1 phenomenology, and it was used for precision tests of the SM in final LEP measurements [1]. It was carefully studied how it can be applied not only in $2 \rightarrow 2$ kinematical configurations but also for configurations with additional hard photons and extended to the picture of exclusive exponentiation [10]. That was highly non-trivial goal, which required massive investment in details of spin amplitudes of multi-photon configurations. Some concepts are explained in Appendix A. Unfortunately such goals are far more demanding for higher than LEP 1 energies and for $p p$ collisions. On the other hand, in many cases it can be avoided, simplifications introduced, and as consequence, an increase in theoretical uncertainties still remains acceptable. 

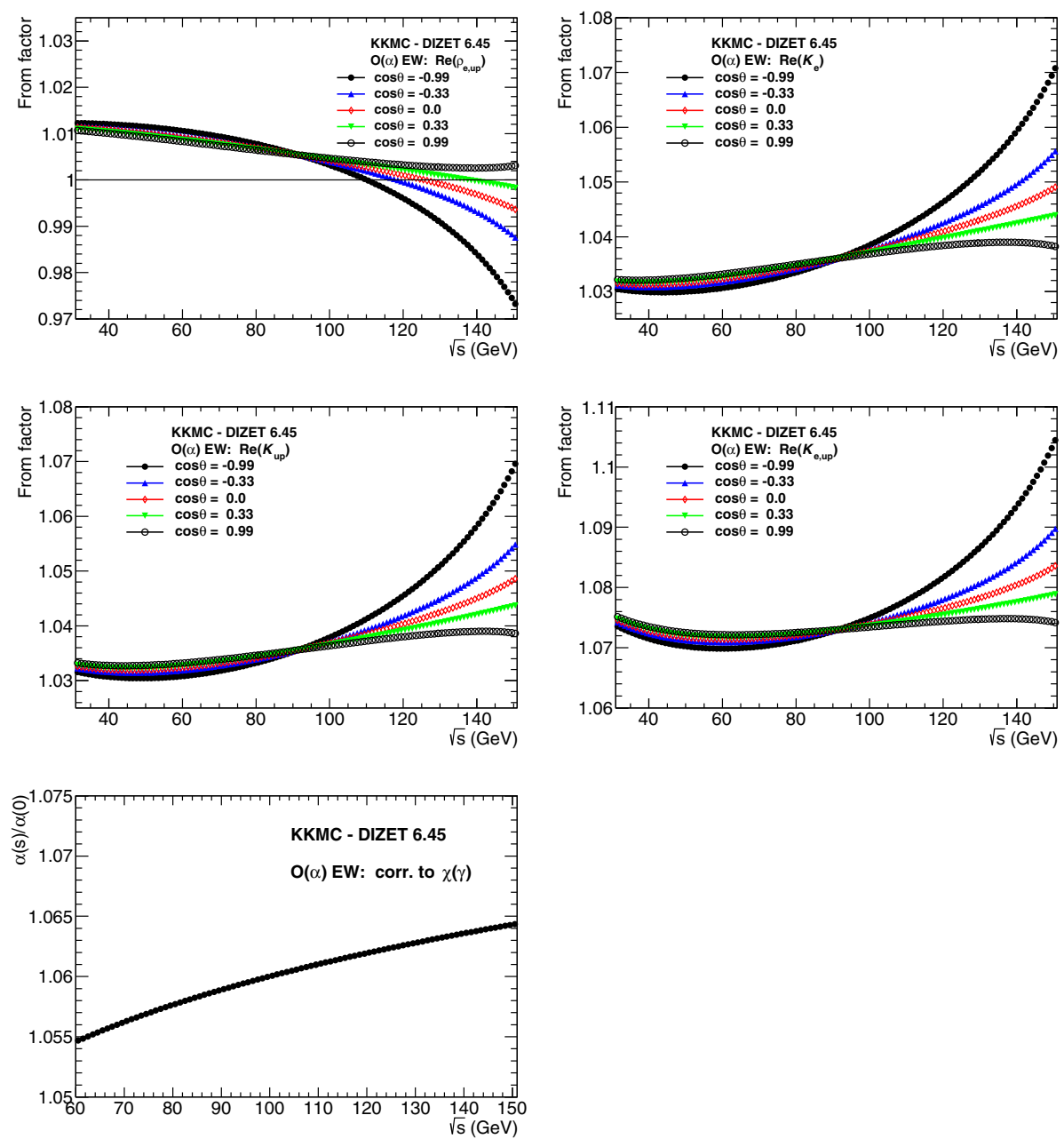

Fig. 1 Plots from the new examples / Di zet-example directory of TauSpinner. The real parts of the $\rho_{e, u p}, \mathscr{K}_{e}, \mathscr{K}_{u p}$ and $\mathscr{K}_{e, u p} \mathrm{EW}$ form factors of $e e \rightarrow Z \rightarrow u \bar{u}$ process, as a function of $\sqrt{s}$ and for the few values of $\cos \theta$ Note that $\mathscr{K}_{e, \text { up }}$ depends on the flavour of outgoing quarks. On the last plot (bottom line), the ratio $\alpha(s) / \alpha(0)=\Gamma_{V_{\Pi}}$ is also shown

\section{Factorization requirements for Born amplitudes}

In the general case, in particular for lepton pair production at the LHC and fully differential distributions, the definition and use of the quark $2 \rightarrow 2$ Born-level scattering process as a well established building block for phenomenology, see, for example, classical papers [28-31], or relatively recent review [32], may seem rather crude and difficult to control approximation, if high precision is required. Let us recall arguments and practical aspects of strong interaction calculations used for TauSpinner design, why it is not necessarily the case.

Properties of the Born-level spin amplitudes lead to features necessary for its factorization from complete formulae including initial or final state QED and/or strong interactions. Quality of such separation is of a decisive importance. Already a long time ago [33], even in 
the presence of hard bremsstrahlung photons, the part of amplitudes which corresponds to the Born-level distribution was identified and separated out. This was studied in the context of hadronic kinematic configuration of the $p p$ LHC physics as well $[18,19]$ and for configurations with up to two high $p_{T}$ jets. These factorization properties were expected, thanks to results of [34]. They require that the Born cross section is described by spherical harmonics of the second order. Indeed, the Born cross section for $f \bar{f} \rightarrow Z / \gamma^{*} \rightarrow \ell^{+} \ell^{-}$reads (azimuthal angle dependence can be avoided with appropriate choice of the reference frame):

$$
\begin{aligned}
\frac{d \sigma_{\text {Born }}^{q \bar{q}}}{d \cos \theta}(s, \cos \theta, p)= & \left(1+\cos ^{2} \theta\right) F_{0}(s) \\
& +2 \cos \theta F_{1}(s)-p\left[\left(1+\cos ^{2} \theta\right) F_{2}(s)+2 \cos \theta F_{3}(s)\right],
\end{aligned}
$$

where $p$ denotes polarization of the outgoing leptons and $\theta$ an angle between incoming quark and outgoing lepton in the rest frame of outgoing leptons. For a general orientation of the reference frame, all second-order spherical harmonics in $\theta$ and $\phi$ angles appear. Second-order spherical harmonics are sufficient also when transverse spin effects are taken into account.

The $F_{i}$ read:

$$
\begin{aligned}
F_{0}(s)= & \frac{\pi \alpha^{2}}{2 s}\left[q_{f}^{2} q_{\ell}^{2} \cdot \chi_{\gamma}^{2}(s)+2 \cdot \chi_{\gamma}(s) \operatorname{Re} \chi_{Z}(s) q_{f} q_{\ell} v_{f} v_{\ell}\right. \\
& \left.+\left|\chi_{Z}^{2}(s)\right|^{2}\left(v_{f}^{2}+a_{f}^{2}\right)\left(v_{\ell}^{2}+a_{\ell}^{2}\right)\right], \\
F_{1}(s)= & \frac{\pi \alpha^{2}}{2 s}\left[2 \chi_{\gamma}(s) \operatorname{Re} \chi(s) q_{f} q_{\ell} v_{f} v_{\ell}+\left|\chi^{2}(s)\right|^{2} 2 v_{f} a_{f} 2 v_{\ell} a_{\ell}\right], \\
F_{2}(s)= & \frac{\pi \alpha^{2}}{2 s}\left[2 \chi_{\gamma}(s) \operatorname{Re} \chi(s) q_{f} q_{\ell} v_{f} v_{\ell}+\left|\chi^{2}(s)\right|^{2}\left(v_{f}^{2}+a_{f}^{2}\right) 2 v_{\ell} a_{\ell}\right], \\
F_{3}(s)= & \frac{\pi \alpha^{2}}{2 s}\left[2 \chi_{\gamma}(s) \operatorname{Re} \chi(s) q_{f} q_{\ell} v_{f} v_{\ell}+\left|\chi^{2}(s)\right|^{2}\left(v_{f}^{2}+a_{f}^{2}\right) 2 v_{\ell} a_{\ell}\right],
\end{aligned}
$$

Unfortunately with $s, t$-dependent EW form factors of Eq. (1), the assumption on spherical harmonics decomposition of the second order only does not hold. Inevitably, the approximation needs to be re-checked if it matches the required precision. Checks if approximations do not deteriorate precision sizably can be obtained from semi-analytical calculation or from fits of re-weighted events distributions. For many applications, it is sufficient to realize that an assumption of angle-independent form factors works well at the vicinity of the $Z$ peak. This can be observed for form factors presented in Fig. 1: lines corresponding to distinct scattering angles cross all at about $\sqrt{s}=M_{Z}$. If off peak contributions to the observable of interest are sizable, then checks with figures indicating the range of $\sqrt{s}$ contributing to observables need to be evaluated. To estimate the size, $e^{+} e^{-}$or parton-level total cross sections and asymmetries, like presented later in Fig. 3, may be used.

The $\sin ^{2} \theta_{W}^{e f f}$ should represent a typical observable and/or coupling constant. This is an approximation. Corrections presented briefly in Appendix A in particular due to box diagram contributions bring not only $s$ but also $t$ (scattering angle) dependence. Figure 2 of $\sqrt{s}$ and flavour-dependent (but with $t$ dependence averaged)

$$
\begin{gathered}
\sin ^{2} \theta_{W}^{\text {eff }}(s)=s_{W}^{2}<\operatorname{Re}\left(K^{f}(s, t)\right)>_{t}, \\
\sin ^{2} \theta_{W}^{\text {eff } f}(s)=s_{W}^{2}<\operatorname{Re}\left(K^{f}(s, t)\right)>_{t},
\end{gathered}
$$

provides a hint on the size of the approximation. The $\sin ^{2} \theta_{W}^{e f f}$ (no $s$ dependence) is supposed to provide the ratio of couplings at the $Z$-pole (averaged over $t$ ) $v_{f}^{\text {eff }} / a_{f}^{\text {eff }}=1-4 q_{f} \sin ^{2} \theta_{W}^{e f f} f$, 
Fig. 2 Averaged for

$p p \rightarrow Z j, Z \rightarrow l \bar{l}$ events

effective weak mixing angles

$\sin ^{2} \theta_{W}^{f \text { eff }}(s)=\operatorname{Re}\left(K^{f}(s, t)\right) s_{W}^{2}$

as a function of $\sqrt{s}$ and

$t$-dependence integrated over,

without (top plot) and with

(bottom plot) box corrections.

The $\mathscr{K}^{f}(s, t)$ form factor

calculated using DIZET 6.45

library and on-mass-shell

$s_{W}^{2}=0.22340108$ were used (see

Table 4). The complete formula

reads $\sin ^{2} \theta_{W}^{\text {eff }}(s, t)=$

$\left.K^{f}(s, t)\right) s_{W}^{2}+I_{f}^{2}(s, t)$. Real

part of $K^{f}(s, t)$ is used, and

imaginary part and $I_{f}^{2}(s, t)$ are

only about $10^{-4}$
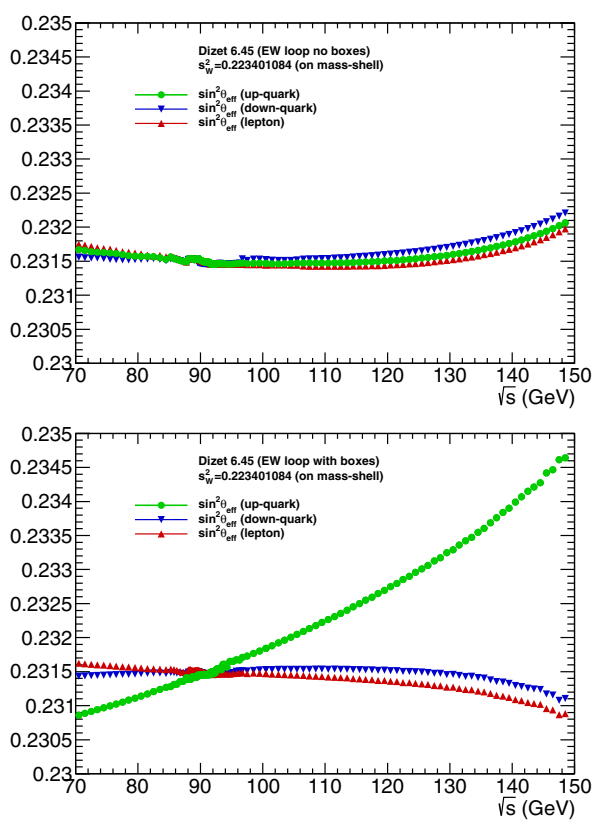

and flavour-dependent variant is a natural extension. For $t$-dependence, EW boxes contribute with correction of isospin-dependent sign. On the other hand, the $\sin ^{2} \theta_{W}^{e f f}(s)$ variations remain below $20 \cdot 10^{-5}$ in the range of $M_{Z} \pm 5 \mathrm{GeV}$. It is known since long that the $t$-dependence originating from $W W$ and $Z Z$ boxes becomes sizable only once $s$ approaches $4 M_{W}^{2}$, the $W$-pair production threshold.

Note that even if for a given observable the nearby of the $Z$ peak dominates for LEP, this may not be the case for LHC or FCC-hh. There, off $Z$ peak contributions are larger due to PDF or beamstrahlung spread. This needs to be kept in mind, in the context of the $\sin ^{2} \theta_{W}^{e f f}$ interpretation as universal pseudo-observable. We recall that off the $Z$ peak form factors dependence on flavour and scattering angle increase, see Fig. 1. It is therefore of interest to validate the range where form-factor angle and energy dependence can be safely ignored and the Effective Born used.

\subsection{Improved Born approximation and effective Born}

From the perspective of calculation of strong interactions, it is convenient to encapsulate all electroweak effects into external currents. Unfortunately, it is not possible to absorb the effects of EW form factors of Eq. (1) (which are all complex and angle/energy dependent) into re-scaling of constants. In particular, definition of $\sin ^{2} \theta_{W}^{e f f}$ would need to be not only flavour, but also observable dependent, invalidating its purpose. Let us now recall details of Effective Born amplitude definition, which differs from formula (1): the form factors are replaced by the effective coupling constants (see also Table 13 of Appendix D).

$$
\begin{aligned}
M E_{\text {Born-eff }}= & \mathcal{N} \frac{\alpha}{s}\left\{\left[\bar{u} \gamma^{\mu} v g_{\mu \nu} \bar{v} \gamma^{\nu} u\right] \cdot\left(q_{e} \cdot q_{f}\right) \cdot \Gamma_{V_{\Pi}} \cdot \chi_{\gamma}(s)\right. \\
& +\left[\bar{u} \gamma^{\mu} v g_{\mu \nu} \bar{v} \gamma^{v} u \cdot\left(v_{e} \cdot v_{f} \cdot v v_{e f}\right)\right.
\end{aligned}
$$




$$
\begin{aligned}
& +\bar{u} \gamma^{\mu} v g_{\mu \nu} \bar{v} \gamma^{v} \gamma^{5} u \cdot\left(v_{e} \cdot a_{f}\right) \\
& +\bar{u} \gamma^{\mu} \gamma^{5} v g_{\mu \nu} \bar{v} \gamma^{v} u \cdot\left(a_{e} \cdot v_{f}\right) \\
& \left.\left.+\bar{u} \gamma^{\mu} \gamma^{5} v g_{\mu \nu} \bar{v} \gamma^{v} \gamma^{5} u \cdot\left(a_{e} \cdot a_{f}\right)\right] \cdot Z_{V_{\Pi}} \cdot \chi_{Z}(s)\right\} \\
v_{e}= & \left(2 \cdot T_{3}^{e}-4 \cdot q_{e} \cdot s_{W}^{2}\right) / \Delta \\
v_{f}= & \left(2 \cdot T_{3}^{f}-4 \cdot q_{f} \cdot s_{W}^{2}\right) / \Delta \\
a_{e}= & \left(2 \cdot T_{3}^{e}\right) / \Delta, \quad \Delta=4 s_{w} c_{w}, \quad a_{f}=\left(2 \cdot T_{3}^{f}\right) / \Delta, \\
\chi_{Z}(s)= & \frac{G_{\mu} \cdot M_{Z}^{2} \cdot \Delta^{2}}{\sqrt{2} \cdot 8 \pi \cdot \alpha} \cdot \frac{s-M_{Z}^{2}+i \cdot \Gamma_{Z} \cdot s / M_{Z}}{s \pi}, \\
\Gamma_{V_{\Pi}}= & 1, \quad Z_{V_{\Pi}}=\operatorname{Re} \rho_{\ell f}\left(M_{Z}^{2}\right), \quad \chi_{\gamma}(s)=1, \\
v v_{e f}= & 1, \quad s_{W}^{2}=\left(1-c_{W}^{2}\right)=\sin ^{2} \theta_{W}^{e f f}\left(M_{Z}^{2}\right), \\
\alpha= & \alpha\left(M_{Z}^{2}\right)=\frac{\alpha(0)}{2-\left(1+\operatorname{Re}_{\gamma \gamma}\left(M_{Z}^{2}\right)\right)} .
\end{aligned}
$$

Note that $\sin ^{2} \theta_{W}^{e f f}\left(M_{Z}^{2}\right), \rho_{\ell f}\left(M_{Z}^{2}\right)$ and $\alpha\left(M_{Z}^{2}\right)$ should now be understood as independent constants, even if in practice calculated in on-mass-shell OMS scheme, see Appendix B. They absorb dominant parts of EW corrections: EW form factors and vacuum polarization corrections. This useful approximation may take into account bulk of the EW effects, and couplings of fixed values are used. There is some level of uncertainty in the numerical values. The best match to Improved Born should correspond to the values predicted by these calculations. In particular, $\sin ^{2} \theta_{W}^{e f f}\left(M_{Z}^{2}\right)=\operatorname{Re} K\left(M_{Z}^{2},-M_{Z}^{2} / 2\right) s_{W}^{2}, s_{W}^{2}=1-M_{W}^{2} / M_{Z}^{2}$, where $M_{W}$ is a calculated quantity including EW corrections, Eq. (6). The $s=M_{Z}^{2}, t=$ $-M_{Z}^{2} / 2$, corresponds to the Born level with scattering angle $\theta=0$. Alternatively, one can use best measured values of Refs. [1,2], and Born expression, ignoring SM constraints, see Table 1.

Once electroweak form factors are replaced with constants, complications due to mixed EW and strong interaction terms in Feynman diagrams, i.e. gauge dependence noncancellation, can be avoided. See Appendix A for some explanation. This may be of importance for calculations which are focused on strong interactions. The price is precision limitation of EW part with respect to Improved Born. Such an approach was used for the previous Tauola/Tauspinner matrix element (ME) implementation [21], with $\sin ^{2} \theta_{W}^{e f f}\left(M_{Z}^{2}\right)$, $\alpha\left(M_{Z}^{2}\right)$ as measured at LEP [2] and $\rho_{\ell f}=1$ for simplification.

To monitor EW effects, we use test observables: $e^{+} e^{-}$(or $q \bar{q}$ ) to pair of leptons, partonlevel cross section $\sigma^{\text {tot }}$, forward-backward asymmetry $A_{F B}$ and $\tau$ lepton polarization $P_{\tau}$, as a function of $\sqrt{s}$.

The $Z / \gamma^{*}->e^{+} e^{-}$(or $\left.q \bar{q}\right)$ cross section $\sigma^{\text {tot }}$ in the EW LO, depends only on coupling constants and two parameters $\left(M_{Z}, \Gamma_{z}\right)$. The effect on $\sigma^{\text {tot }}$ from EW loop corrections is due to corrections to the propagators: vacuum polarization corrections (running $\alpha$ ) and $\rho$ form factor. This causes a change in relative contributions of the $Z$ and $\gamma$, and change of the $Z$-boson vector to axial coupling ratio $\left(\sin ^{2} \theta_{W}^{e f f}\right)$. They affect not only $s$-dependence but normalization of the cross section too.

The asymmetry $A_{F B}=\frac{\sigma(\cos \theta>0)-\sigma(\cos \theta<0)}{\sigma(\cos \theta>0)+\sigma(\cos \theta<0)}$ is defined in the standard way. For $e^{+} e^{-}$collision, an angle $\theta$ between incoming particle and outgoing lepton is taken. For $p p$ collision, the Collins-Soper frame [35] is used for angle $\theta$ definition. The asymmetry varies strongly with $\sqrt{s}$ around the $Z$ peak because it is proportional to product of small vector $v_{i}$ and $v_{f}$ 


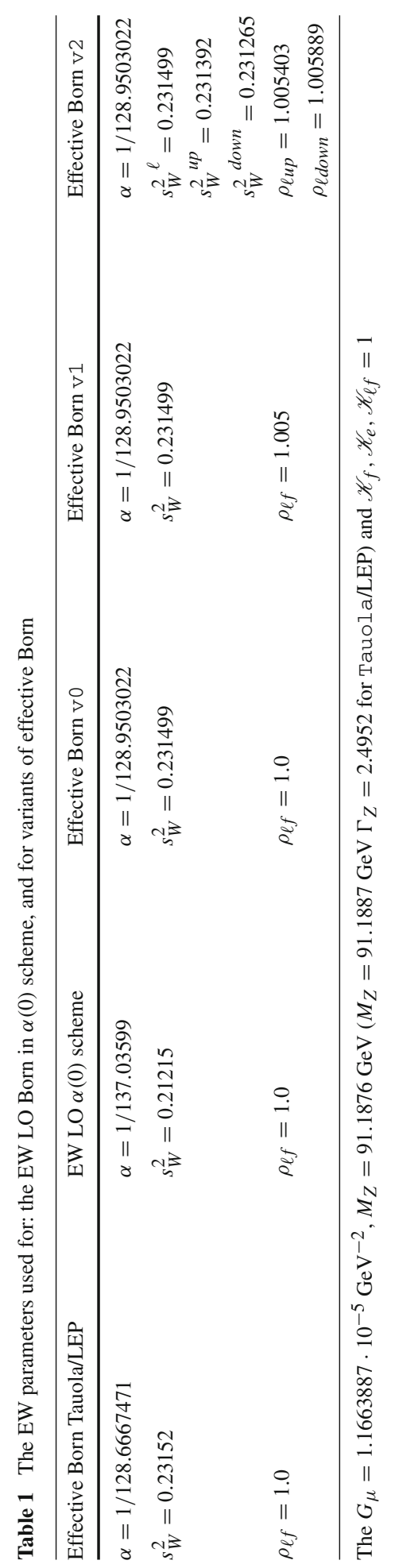


couplings of incoming parton and outgoing lepton. The product is specially small for $e^{+} e^{-}$ initial state. That is why, off the peak, $s$-channel $Z$-photon interference quickly becomes sizable.

The $\tau$ polarization $P_{\tau}=\frac{\sigma(R)-\sigma(L)}{\sigma(R)+\sigma(L)}$, where $\sigma(R / L)$ denote cross section for production of right/left hand polarized $\tau$, is of interest in itself as it offers independent data point for precision EW sector measurements. It is of convenience, because, in the first approximation, it is linearly proportional to $\sin ^{2} \theta_{W}^{e f f}$, and thus, it is useful for discussion of systematic uncertainties. The systematic errors for this measurement differ from that of $\sigma^{\text {tot }}$ or $A_{F B}$, predominantly because $P_{\tau}$ is not measured directly, but through distribution of $\tau$ decay products only. These points were recently recalled in [36]. On the other hand, relation between $P_{\tau}, Z$ couplings and $\sin ^{2} \theta_{W}^{e f f}$ is not affected and generally is of the same nature like for $A_{F B}$. That is why this data point is particularly suitable for discussion with $e^{+} e^{-}$semi-analytical results.

Test observables and $\sin ^{2} \theta_{W}^{e f f}$. It is worth to point out that of the $e^{+} e^{-}$scattering results, the ones for the $P_{\tau}$ are particularly convenient in discussion of $\sin ^{2} \theta_{W}^{e f f}$. This is because $P_{\tau}$ is linearly proportional to small vector $Z$-lepton coupling, thus to $\sin ^{2} \theta_{W}^{\text {eff }}$ itself. Also, the $P_{\tau}$ varies with energy in the vicinity of the $Z$ peak relatively slowly. To a good approximation, as one can easily deduce from formula (5) any variation $\delta$ of $P_{\tau}$ measured at the $Z$ peak translates into $\sim \frac{1}{8} \delta$ shift ${ }^{2}$ of $\sin ^{2} \theta_{W}^{\text {eff }}$ quite independently of the flavour of incoming state. This holds not only for $e^{+} e^{-}$but for incoming quarks too. For $A_{F B}$ and $\sigma^{\text {tot }}$, similar relations can be obtained, and then, $\rho$ and $\alpha$ dependence would need to be taken into the picture. The initial state flavour and much stronger energy dependence would lead to multitude of cases. That is why we will use $P_{\tau}$ as an example to discuss suitability of the $\sin ^{2} \theta_{W}^{e f f}$ picture and its limitations.

In Fig. 3, our test observables are shown for the $e^{+} e^{-}$case, to start numerical comparisons of Improved Born and Effective Born. Tauola/LEP initialization of Effective Born, as specified in Table 1 and installed in Tauola distribution, is shown. Differences are not large, but possibly not always satisfactory for precision physics. In fact Tauola/LEP Effective Born becomes insufficient for high precision measurements, especially of hadron colliders (where off the $Z$ peak contributions, contrary to the $e^{+} e^{-}$circular colliders, cannot be minimized/excluded by the fixed colliding quark energies).

\section{From Improved Born to Effective Born: numerical results}

Let us now attempt to identify those Effective Born simplifications which are of numerical consequences and those which are important from the theoretical perspective, but hopefully not so much numerically. From theoretical perspective, use of electroweak form factors together with higher-order QED or QCD corrections is cumbersome because of imaginary parts and energy and scattering angle dependence, which make it impossible or at best complicated to absorb them into redefinition of coupling constants. The $\mathscr{K}_{e, f}$ affect couplings, whereas $\rho_{\ell f}(s, t)$ and $\Pi_{\gamma \gamma}(s)$ contribute overall factors for $\mathrm{Z}$ boson and photon exchange amplitudes. Imaginary parts can be thus absorbed partly, into $M E$ overall phase. The real parts in the redefinition of the overall couplings size are used. One may want to underline in this context the "LEP2005 style" choice of Improved Born simplification, where imaginary

At the $Z$ peak $P_{\tau} \simeq \frac{2 v_{e}}{a_{e}}$. 

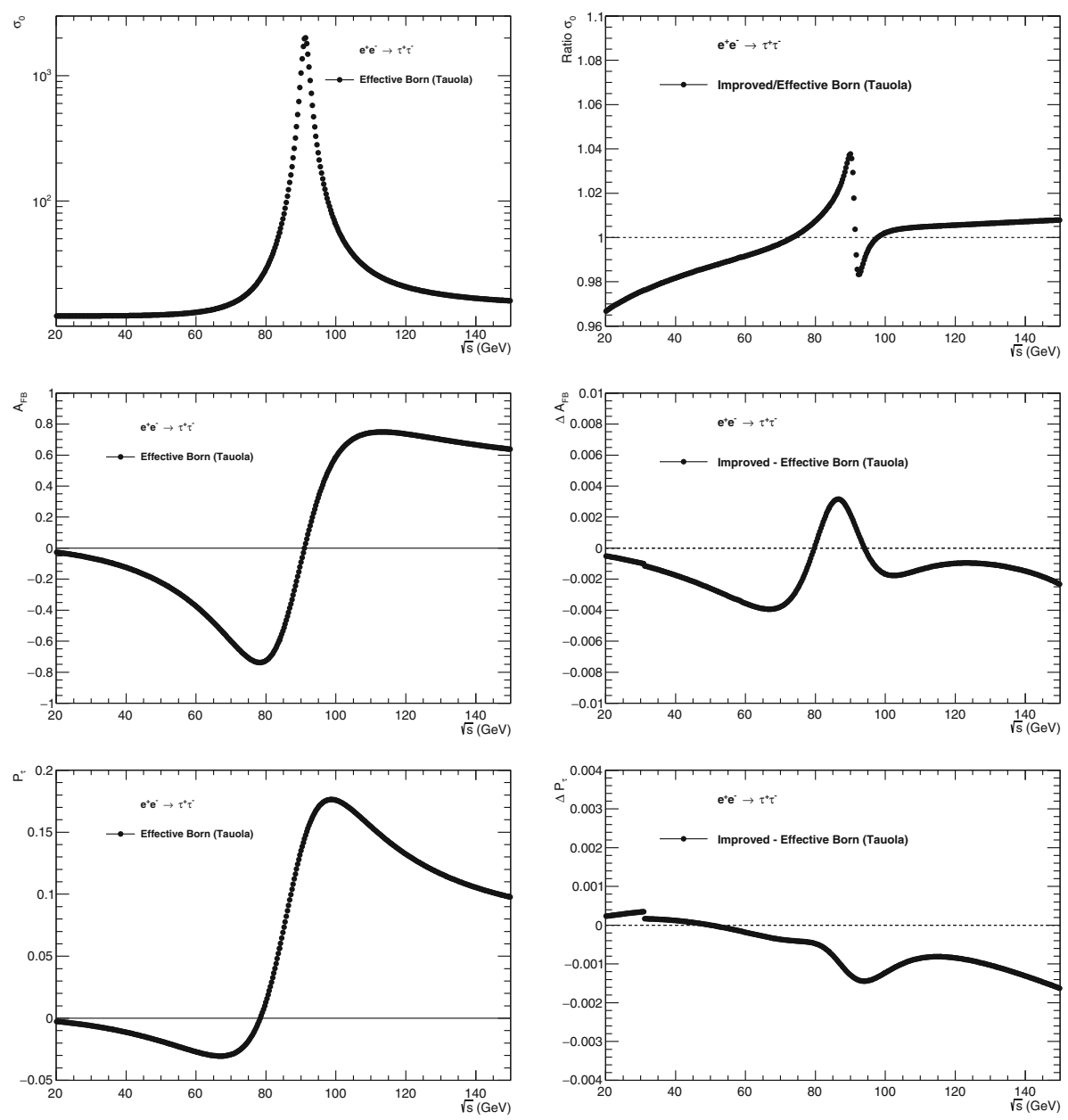

Fig. 3 The $\sigma^{\text {tot }}(s), A_{F B}(s)$ and $P_{\tau}(s)$ (left side, top, centre and bottom plots, respectively) of TauSpinner calculation with Effective Born (Tauola/LEP as installed in Tauola since December 2019). In the right side plots, results of Improved Born calculations with EW form factors from DIZET 6.45 are compared with those of the left side plots. Note the differences depicted in the right side plots are enhanced in part because input parameters of Tauola/LEP initialization, see Table 1 for details. The $M_{z}$ and $\Gamma_{Z}$ are slightly different than those used for Improved Born, causing sizable kink in the top right plot of $\sigma^{\text {tot }}$. On the right-hand side plots, there are minor discontinuities at $30 \mathrm{GeV}$, too. They are smaller than calculation precision and are due to granularity used for tabulation dominated

part of $\Pi_{\gamma \gamma}(s)$ is not ignored, whereas usually it is the case especially if energy-dependent $\alpha_{Q E D}(s)$ is used.

In the following, the results of consecutive simplifications will be compared to Improved Born results, which are the most precise ones.

We keep all input parameters as of Improved Born but gradually simplify EW correcting terms. For reference results, Improved Born and DIZET 6.45 EW library were used.

The aim is to identify those simplifications which are numerically of small consequences, but may represent an obstacle to install electroweak interaction as external current in multiloop QCD calculations. 

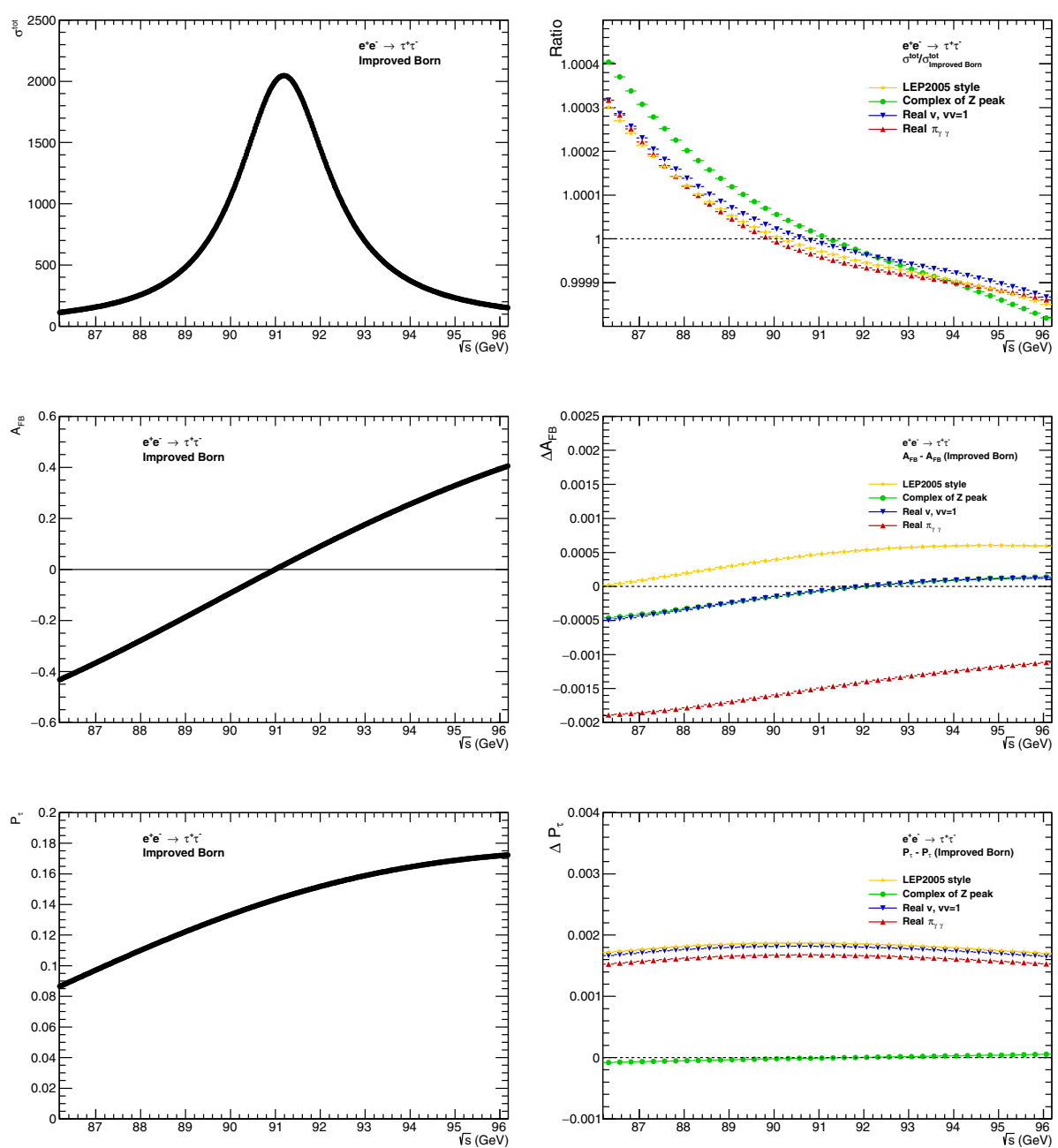

Fig. 4 Left side plots, Improved Born-level and vicinity of the $Z$ peak: $\sigma^{\text {tot }}$ (top), $A_{F B}$ (middle) and $P_{\tau}$ (bottom). Right side plots enumerate with ratios or differences the effects of simplifications with respect to Improved Born results. Green points: instead of form factors, their constant values calculated at $s=M_{Z}^{2}$, $t=-M_{Z}^{2} / 2$ are used. Blue triangles: as for green ones, but in addition $v v_{\ell} f=1$ and only real parts of $v_{e}$, $v_{f}$ are used. Red triangles: as in blue triangles, but only real parts of $\Pi_{\gamma \gamma}$ and $\rho_{\ell f}$ are taken into account. Yellow stars: with respect to red triangles imaginary parts of $\Pi_{\gamma \gamma}$, are switched back on

We test the impact of simplification steps from Improved $\rightarrow$ Effective Born, on $\sigma^{\text {tot }}, A_{F B}$ and $P_{\tau}$ and for all the elementary processes: $e^{+} e^{-} \rightarrow \tau^{+} \tau^{-}$and $u \bar{u}(d \bar{d}) \rightarrow \tau^{+} \tau^{-}$. We concentrate first on $e^{+} e^{-} \rightarrow \tau^{+} \tau^{-}$process and choose for Figs. 4 and 5 the energy range important for the measurement of the $Z$ boson couplings, that is $M_{Z} \pm 5 \mathrm{GeV}$. The $e^{+} e^{-}$case is simpler to present, and conclusions would not differ much if instead quark-level processes would be used.

Approximation as of green points in Fig. 4 (marked "complex of $Z$ peak"). In this first step of approximation introduced into Eq. (1), the $s$ - and $t$-dependent form factors are replaced with their values at the $Z$ peak and for the scattering angle $\cos \theta=0$. 
One can see that if constant complex couplings calculated at the $Z$ peak instead of $s, t$ dependent form factors are used, in the range of $M_{Z} \pm 5 \mathrm{GeV}$ the $P_{\tau}, A_{F B}$ and $\sigma^{\text {tot }}$ depart from the exact result at the peak, respectively, by up to $8 \cdot 10^{-5}, 45 \cdot 10^{-5}$ and $40 \cdot 10^{-5}$. These largest differences are at the edge of the range, where cross section is already about a factor 20 smaller than at the peak.

If in addition $v v_{e f}$ was set to 1, additional changes are marginal. That is why in the figure the case of $v v_{\ell f}=1$ is not presented. Once $v v_{\ell f}=1$ is set, mixing term is avoided and effective couplings are attributed separately to incoming and outgoing flavour.

Approximation as of blue triangles in Fig. 4 (marked as "real $v, v v=1$ "). The second step is to neglect imaginary parts of the vector couplings to $Z$. They are about factor of 100 smaller than the real parts. Now, the difference became larger for $P_{\tau}\left(166 \cdot 10^{-5}\right)$, but similar $\left(50 \cdot 10^{-5}\right.$ and $32 \cdot 10^{-5}$ ) for $A_{F B}$ and $\sigma^{\text {tot }}$. That means non-negligible degradation of precision for $P_{\tau}$ evaluation, corresponding, in the language of $\sin ^{2} \theta_{W}^{\text {eff }}$ to a $21 \cdot 10^{-5}$ prediction uncertainty. Approximations as of red triangles and yellow stars in Fig. 4 (marked, respectively, " real $\Pi_{\gamma \gamma}$ " and "LEP2005 style"). The role of the imaginary part of $\Pi_{\gamma \gamma}$ requires special attention, particularly for $A_{F B}$. For red triangles, with respect to previous case, imaginary parts of $\Pi_{\gamma \gamma}$ and $\rho_{\ell f}$ are set to zero, whereas for yellow stars, the imaginary part of $\rho_{\ell f}$ is set to zero only. The differences for "real $\Pi_{\gamma \gamma}$ " ("LEP 2005 style") are, respectively, $152 \cdot 10^{-5}, 188 \cdot 10^{-5}$, $32 \cdot 10^{-5},\left(172 \cdot 10^{-5}, 60 \cdot 10^{-5}, 30 \cdot 10^{-5}\right)$. The numerical effect of these imaginary parts, which cannot be easily absorbed in re-definition of the couplings, needs to be kept in mind. With "LEP2005 style" parametrization, we still do not address more subtle LEP time choices used in data analysis. In particular, of parametrizations used to separate contributions from s-channel exchange of $Z$ boson and virtual photon exchange interfering background.

In practice, in "LEP2005 style" variant, we use formula (1) but with $K_{e / f}(s, t) \rightarrow$ $\operatorname{Re} K_{e / f}\left(M_{Z}^{2},-M_{Z}^{2} / 2\right), K_{e f}(s, t) \rightarrow 1, \rho_{\ell f} \rightarrow \operatorname{Re} \rho_{\ell f}\left(M_{Z}^{2}\right)$ that translates into use of flavour-dependent $\sin ^{2} \theta_{W}^{e / f}$ eff $\left(M_{Z}^{2}\right)$. For $\alpha\left(M_{Z}^{2}\right)$, the replacement $\Pi_{\gamma \gamma}(s) \rightarrow \Pi_{\gamma \gamma}\left(M_{Z}^{2}\right)$ with a complex value is used. Now, the purpose is to evaluate numerical consequences of $\alpha(s)$ 's imaginary part. But it is also similar to what was used at a time of final precision data analysis of all LEP collaborations combined [2]. Motivation of the choices are presented in Ref. [15] and their numerical impact in Table 19 there. See also Section 5.4.4 of [2].

Four initialization variants are used in Fig. 4 and eleven ones in Table 14 of Appendix D. Those eleven initializations are detailed in Table 12 and can be activated with the KEYGSW parameter of TauSpinner, to enable user to evaluate numerically simplifications of Improved Born EW. In fact, a lot of our numerical results were obtained with the help of TauSpinner package semi-analytic scripts, described in Appendix C. Initialization variants used for Fig. 4 are less straightforward to activate they may require minor changes in the TauSpinner code. These results were useful for the discussion, but supposedly of less interest for TauSpinner users.

Main observations (i) The $\Pi_{\gamma \gamma}$ imaginary part, formally contributing at higher orders, was included in calculations for final LEP time data analysis. Its impact is largest for $A_{F B}$, whereas for $P_{\tau}$ imaginary parts of $v_{e}, v_{f}$ couplings are more important. (ii) The form-factor replacement with constant effective couplings is numerically less important than when their imaginary parts are dropped. Also, the closer to the $Z$ peak one goes, the smaller the disturbing of the Effective Born picture from photon exchanges. ${ }^{3}$ The same is true for the complex part of $\rho_{\ell f}$. This is because more realistic observables usually tend to average contributions

3 This is because at the peak $Z$ propagator $1 /\left(s-M_{Z}^{2}+i \Gamma_{Z} M_{Z}\right)$ is much larger than the one of photon: $1 / M_{Z}^{2}$. Variation of the propagator phase leads to a partial cancellation of form-factor imaginary parts in $Z-\gamma$ exchange interference contributions. 
from the below and above $Z$ peak. Generally speaking, numerical impact on observables is not universal and distinct sets of effective couplings might be needed for each of our test observables to match best the result of the Improved Born approximation.

\subsection{The $\mathrm{v} 0, \mathrm{v} 1, \mathrm{v} 2$ variants of Effective Born}

The formulae for Improved Born Eq. (1) and for Effective Born Eq. (5) differ with subtle, but numerically important details. We evaluate them with the help of options in Tauspinner explained in Appendix D, in particular in Table 12.

One can ask the question how close can one approach Improved Born results, with the effective ones, without breaking cancellations, mentioned in Appendix A, necessary for strong interaction or QED ISR calculations. Let us introduce few variants:

- The v0 variant is using formula (5) for spin amplitude, with $\alpha(s)=\alpha\left(M_{Z}^{2}\right), s_{W}^{2}=$ $\sin ^{2} \theta_{W}^{e f f}\left(M_{Z}^{2}\right)$, but with $\rho_{\ell f}=1.0$.

- The $\mathrm{v} 1$ variant is using formula (5) for spin amplitude, parameters are set as for $\mathrm{v} 0$ parametrization, but $\rho_{\ell f} \neq 1$.

- The v2 variant is using formula (5) for spin amplitude, and parameters are set as if both $s_{W}^{2}$ and $\rho_{\ell f}$ were flavour-dependent, and equal at the $Z$-pole to the Dizet 6.45 predicted ones. See Tables 1 and 4.

- The Tauola/LEP variant differs from v0 by numerical values of $\alpha, s_{W}^{2}=\sin ^{2} \theta_{W}^{e f f}\left(M_{Z}^{2}\right)$. Also, $M_{Z}$ and $\Gamma_{Z}$ differ, see Table 1. It is worth to point that Eq. (5) remains the same as for the Effective Born used at LEP 1 times.

The consecutive three variants $\mathrm{v} 0, \mathrm{v} 1, \mathrm{v} 2$ of Effective Born are ordered with increasing complexity and improving precision to reproduce the Improved Born results, see Table 1. Note that depending on the activated variant, numerical values for $\sin ^{2} \theta_{W}^{e f f}, \rho_{\ell f}$ and $\alpha$ differ, to optimize results for our test observables. The flavour dependence for $\sin ^{2} \theta_{W}^{e f f}$ and $\rho_{\ell f}$ may appear, too.

Details of listed above variants for Effective Born are provided in Table 1. Nonessential details are delegated to Appendix B. For completeness the reference starting points, Tauola/LEP parametrization and the EW LO Born parametrization in EW $\alpha(0)$ scheme are provided.

Let us now present numerical results. Figure 5 is similar to Fig. 4 but serves different purposes. It enumerates the performance of $\mathrm{v} 0, \mathrm{v} 1, \mathrm{v} 2$ Effective Born with respect to the Improved Born. The shifts of $\mathrm{v} 0$ with respect to Improved Born, respectively, for $P_{\tau}$, $A_{F B}$ and $\sigma^{\text {tot }} \mathrm{read} 140 \cdot 10^{-5}, 370 \cdot 10^{-5}$ and $1000 \cdot 10^{-5}$. This is already substantially better than EW at LO. ${ }^{4}$ Obviously, coefficient in front of the $Z$ exchange propagator needs to be corrected further: if $\rho_{\ell f}$ is used of variant $\mathrm{v} 1$, differences with respect to Improved Born reduce to, respectively, $140 \cdot 10^{-5}, 200 \cdot 10^{-5}$ and $50 \cdot 10^{-5}$. With the $\mathrm{v} 2$ setting, re-installing flavour dependence of couplings, we obtain results for the Effective Born which differ from the EW corrected ones by $140(40) \cdot 10^{-5}, 200(40) \cdot 10^{-5}$ and $60(20) \cdot 10^{-5}$. Numbers in brackets were obtained with virtual $\gamma$ contribution switched off. ${ }^{5}$

We have presented numerical results useful to evaluate robustness of the Effective Born picture, where effective couplings are used for describing EW effects, and compare it with

4 If Effective Born v0 would be used, but with LO EW parameters the shifts on our tests observables with respect to Improved Born would be about a factor 100 larger.

5 This hints that Effective Born may work better for $p p$ collisions than for $e^{+} e^{-}$, because of smaller electric charge of quarks than of leptons. 

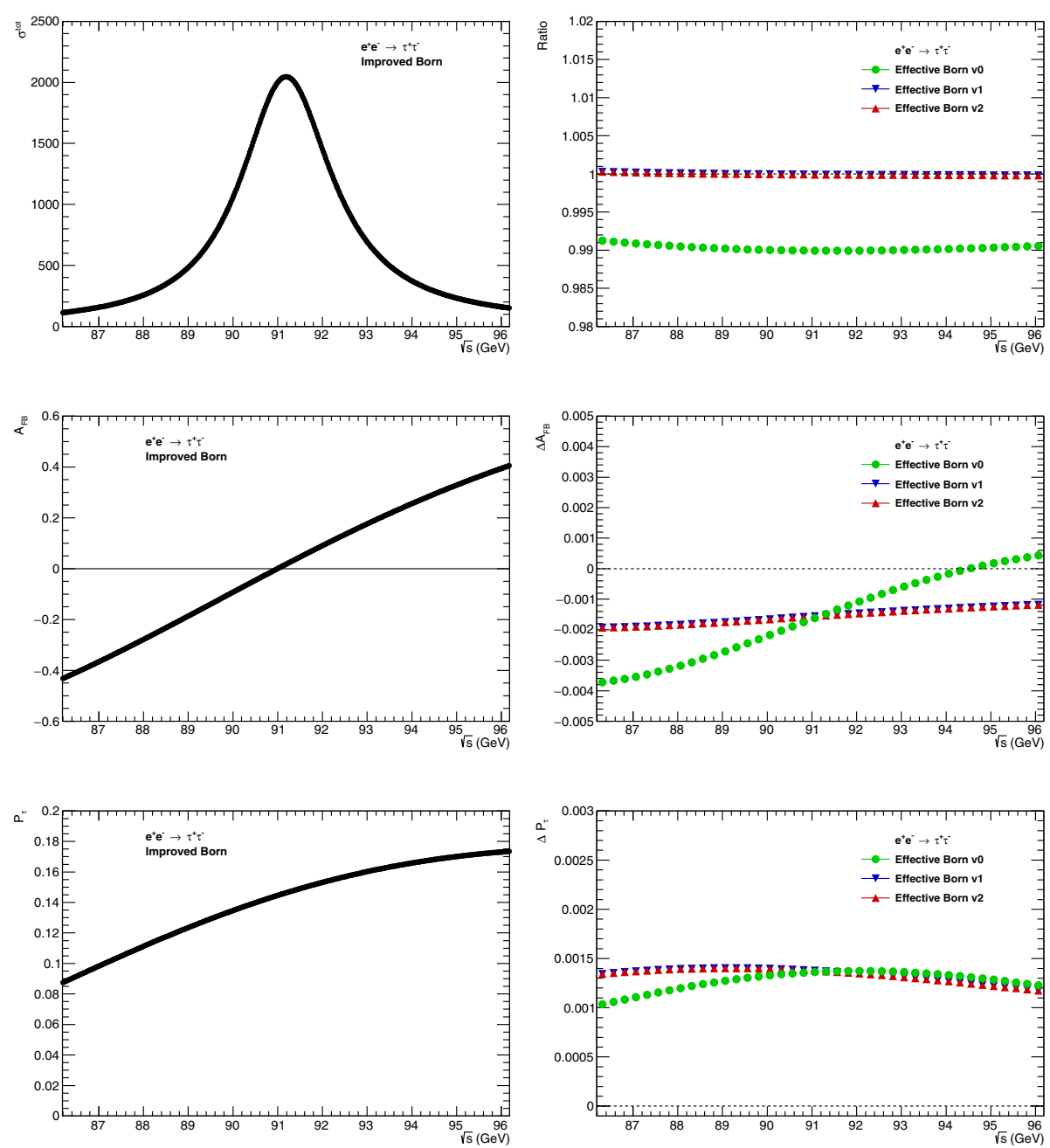

Fig. 5 Left side plots, Improved Born in the vicinity of the $Z$ peak: $\sigma^{\text {tot }}$ (top), $A_{F B}$ (middle) and $P_{\tau}$ (bottom), as in Fig. 4. Right side plots enumerate with ratios or differences the effects of Effective Born simplifications with respect to Improved Born. Green points: Effective Born v0, blue triangles: Effective Born v1, red (rotated) triangles: Effective Born v2

results of the Improved Born picture. We will use Improved Born predictions as a reference for $e^{+} e^{-}$and $q \bar{q}$ cases. ${ }^{6}$ As we see, Effective Born v2 variant works quite well around the $Z$ pole, for the line shape and forward-backward asymmetry too. It may be not as straightforward to implement into strong interaction Monte Carlo programs as is the case of $\mathrm{v} 0$ or $\mathrm{v} 1$.

\footnotetext{
6 In Appendix, Table 14, two versions of the Improved Born are used, with weak boxes included and not. This is important for large energy ranges. For $e^{+} e^{-}$, we concentrate mostly on the region of the $Z$ pole where the impact of EW boxes is marginal. We demonstrate the quantitative impact of the $s, t$-dependence, which cannot be absorbed into effective couplings.
} 
The Effective Born variants differ from Improved Born; constants instead of form factors are used. This is partly compensated with adjustments of input parameters. It is interesting to evaluate limits of $\sin ^{2} \theta_{W}^{e f f}$ in interpretation of the $Z-l-l$ coupling measurements.

From these results, particularly for $P_{\tau}$, we may conclude that approaches that rely on effective couplings may not work well for the $\sin ^{2} \theta_{W}^{\text {eff }}$ precision tag up to about $20 \cdot 10^{-5}$. For further improvement, revisiting EW effects in their complexity is required. Use of numerically adapted Eq. (5) constants, which originally in Eq. (1) were multiplied by form factors, does not suffice. For high precision, the picture of effective couplings is not universal: the choice appropriate for $A_{F B}$ may not be optimal for $P_{\tau}$.

\subsection{Case of $p p \rightarrow l l$ processes at LHC}

Let us now discuss properties of these test observables distributions in the $p p$ case. For $p p$ collisions, convolution with parton distribution functions would make interpretation of numerical results for our test observables $\sigma^{t o t}, A_{F B}$ and $P_{\tau}$ obscured, or unrealistic if dropped out.

In Fig. 6 (top), distributions of generated and EW corrected Z-line shape (through $\sigma^{\text {tot }}$ ) are shown. For the EW weight calculation, $\cos \theta$ is needed. Because of parton distribution and jet presence, reconstruction is needed and $\cos \theta^{*}$ definition of the scattering angle as defined in [22] is used. On the logarithmic scale, the difference is barely visible. In the following plots of the same figure, we study it in more details. The ratios of the $Z$ lineshape distributions with gradually introduced EW corrections are shown. We evaluate the size of complete Improved Born predictions with respect to variants of Effective Born. For reference predictions (denominator of the weights), the following: (i) EW LO $\alpha(0)$ (middletop plot), (ii) Effective Born v0 (middle-bottom plot) and (iii) Effective Born v2 (bottom plot), are used. For numerators, Improved Born of form factors without/with box diagram contributions are used. At the Z-pole, complete EW corrections of Improved Born give for $\sigma$ about $0.01 \%$ different results from the one of Effective Born v2. It demonstrates that if for event generation an EW LO matrix element is used with effective variant $\mathrm{v} 2$ parametrization, the size of missing EW effects will be significantly reduced.

Similar conclusions can be drawn from Table 2, where the numerical impact of EW corrections on the normalization, i.e. ratios of the $p p$ cross sections integrated in the range $81<m_{e e}<101 \mathrm{GeV}$ and $89<m_{e e}<93 \mathrm{GeV}$, is given. Total EW corrections for EW LO $\alpha(0)$ cross section are about 0.035 , while for the Effective Born $\mathrm{v} 0$ it is of about 0.01 and for Effective Born v2 is of about 0.0001. The main improvement of $\mathrm{v} 2$ with respect to $\mathrm{v} 0$ is thanks to $\rho_{\ell f} \neq 1$ introduced already for $\mathrm{v} 1$.

Let us now turn our attention to the forward-backward asymmetry $A_{F B}$. Again for the $p p \rightarrow Z / \gamma^{*} \rightarrow l^{+} l^{-}$process, energy range from 60 to $150 \mathrm{GeV}$ was chosen which is of interest for EW effects. As in the case of cross section, shape and size of the corrections depend on whether box exchange diagrams are included in the Improved Born form factors. In top plot of Fig. 7, the $A_{F B}$ distribution as generated (EW LO) and superimposed with EW corrected result is shown. The points for the two cases are practically indistinguishable. Further three plots of the figure, with the difference $\Delta A_{F B}=A_{F B}-A_{F B}^{r e f}$, provide details. For the reference $A_{F B}^{r e f}$, the three versions of the Effective Born detailed in Table 1 are used again: (i) EW LO $\alpha(0)$, (ii) $\mathrm{v} 0$ and (iii) $\mathrm{v} 2$, while for $A_{F B}$ Improved Born is used. The EW corrections for $A_{F B}^{r e f}$ of EW LO Born with $\alpha(0)$ scheme, integrated around the $Z$-pole, necessary to reproduce Improved Born result can reach -0.03514 , see Table 3 . The Effective 
Fig. 6 Top: $Z$ line-shape $(\sqrt{s}$ invariant mass of the $l^{+} l^{-}$pair) distribution as generated with Powheg+MiNLO (blue triangles) and after re-weighting introducing all EW corrections discussed (red triangles). The points are barely distinguishable. Ratios of Improved Born results (with and without EW boxes) to Effective Born in: (i) EW LO $\alpha(0)$ scheme are given in upper-middle, (ii) in lower-middle to Effective Born $\mathrm{v} 0$ and (iii) in bottom plots to Effective Born v2
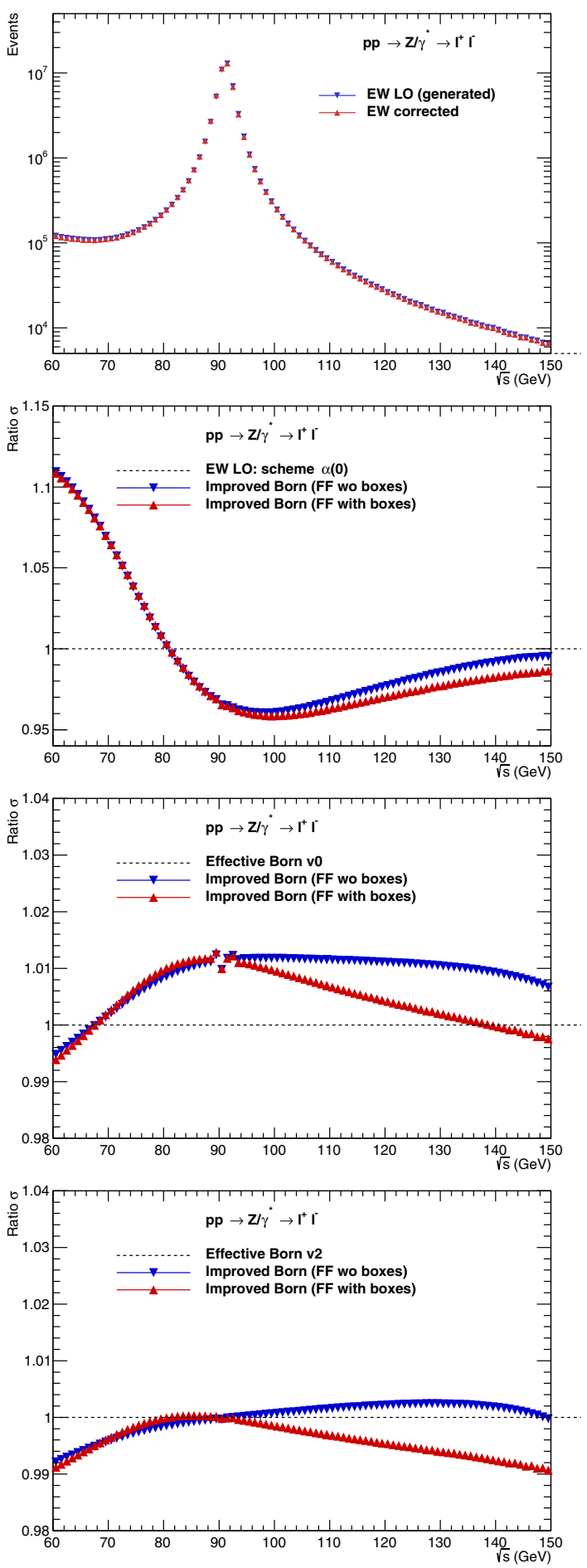
Table 2 EW corrections to cross sections $\sigma^{\text {tot }}$ in the specified mass windows

\begin{tabular}{lll}
\hline Corrections to cross section & $89<m_{e e}<93 \mathrm{GeV}$ & $81<m_{e e}<101 \mathrm{GeV}$ \\
\hline$\sigma^{\text {tot }}$ (Improved Born, no boxes) $/ \sigma(\mathrm{EW} \mathrm{LO} \alpha(0))$ & 0.96505 & 0.96626 \\
$\sigma^{t o t}$ (Improved Born, with boxes) $/ \sigma(\mathrm{EW} \mathrm{LO} \alpha(0))$ & 0.96510 & 0.96631 \\
$\sigma^{\text {tot }}$ (Eff. Born v0) $/ \sigma($ Improved Born, with boxes) & 1.01142 & 1.01135 \\
$\sigma^{\text {tot }}$ (Eff. Born v1) $/ \sigma($ Improved Born, with boxes) & 1.00130 & 1.00132 \\
$\sigma^{\text {tot }(\text { Eff. Born v2) } / \sigma \text { (Improved Born, with boxes) }}$ & 0.99989 & 0.99987 \\
\hline
\end{tabular}

DIZET 6.45 form factors and running width were used in re-weighting of LHC $p p \rightarrow Z j ; Z \rightarrow l^{+} l^{-}$ events simulated at $8 \mathrm{TeV}$. From the first two lines, magnitude of EW corrections with respect to lowest order, $\alpha(0)$ scheme can be read off. Following three lines demonstrate precision of Effective Born variants with respect to Improved Born

Born $v 0$ reproduces Improved Born up to $\Delta A_{F B}$ of about -0.0004 , while the Effective Born $\mathrm{v} 2$ up to -0.0002 . The $\mathrm{v} 2$ variant is again better by a factor of two than the $\mathrm{v} 0$ one.

These results point to limitation at about $20 \cdot 10^{-5}$ for $\sin ^{2} \theta_{W}^{e f f}\left(M_{Z}^{2}\right)$ uncertainty and real constants Effective Born parametrization. This even if $\alpha\left(M_{Z}\right)$ and $\rho_{l f}\left(M_{Z}\right)$ is used. This is not as easy to see as in the case of $P_{\tau}$ when, as pointed in Sect. 4, $\delta P_{\tau} \simeq \delta \sin ^{2} \theta_{W}^{\text {eff }}\left(M_{Z}^{2}\right)$. For $A_{F B}$, this relation is more complex and is also incoming parton flavour dependent.

Even if $P_{\tau}$ is not particularly suitable for $p p$ collision measurements, it weakly depends on the production process and is useful for numerical $\sin ^{2} \theta_{W}^{e f f}\left(M_{Z}^{2}\right)$ uncertainties evaluation in general case. That is why previous subsection results are of the relevance for $p p$ too and are of no need to repeat.

\section{Electroweak corrections in TauSpinner: library versions and initializations}

In the present section, we address the impact of the DIZET library variants, which have by now a life-time of more than three decades. The versions of the DIZET EW correction library, which are used in our numerical discussion, are presented briefly in Appendix F; details are given in Ref. [26]. Specification of initializations is collected in Appendix B.1. One may wonder if the last version would not suffice. However, availability of the software used for the solutions of legacy measurements is of some value. That is why in Ref. [26] several versions of the present and past EW DIZET library were collected. On the other hand, archived with [23] less popular calculations of the past will not receive our attention.

Each of the four versions of DIZET library of EW effects comes with a wealth of options, which may be activated with their input flags. These options can be used to evaluate the importance of the particular improvement introduced over the years. The graphical programs to monitor the changes are available in TAUOLA/TauSpinner / examples directory. The tau-reweight-test. Cxx can be used to demonstrate how events can be corrected with the weight representing improvement from TAUOLA Effective Born of its constant couplings to the one of Improved Born of formula (1) with form factors interpolated from the tables prepared with KKMC interface to DIZET.

The default for anomalous Born function, implemented for the first time in Ref. [37], is not anymore a dummy function but is now the one of EW Improved Born, which uses the EW form-factor tables (if available). The new sub-directory Di zet-example collects programs and scripts for form-factor graphic representation. Plots of form factors can be drawn, as a 
Fig. 7 Top: the $A_{F B}$ distribution ( $\sqrt{s}$ invariant mass of the $l^{+} l^{-}$ pair) as generated in Powheg+MiNLO sample (blue triangles) and after re-weighting introducing all EW corrections (red triangles). The two choices are barely distinguishable. The differences

$\triangle A_{F B}=A_{F B}-A_{F B}^{r e f}$, of Improved Born results (with and without EW boxes) to Effective Born in: (i) EW LO $\alpha(0)$ scheme are given in upper-middle, (ii) in lower-middle to Effective Born v0 and (iii) in bottom plots to Effective Born v2
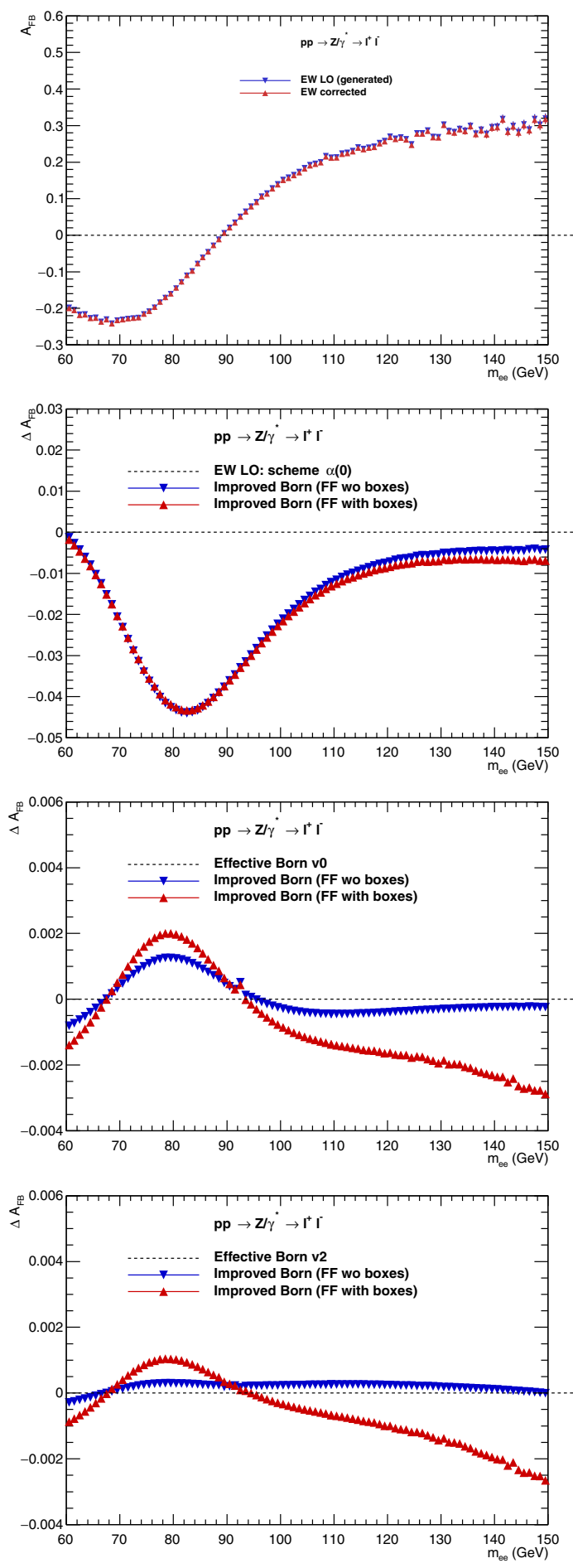
Table 3 The difference in forward-backward asymmetry, $\Delta A_{F B}$, in the specified mass windows

\begin{tabular}{lll}
\hline Corrections to $A_{F B}$ & $89<m_{e e}<93 \mathrm{GeV}$ & $81<m_{e e}<101 \mathrm{GeV}$ \\
\hline$A_{F B}($ Improved Born, no boxes $)-A_{F B}($ EW LO $\alpha(0))$ & -0.03491 & -0.03515 \\
$A_{F B}($ Improved Born, with boxes $)-A_{F B}($ EW LO $\alpha(0))$ & -0.03489 & -0.03514 \\
$A_{F B}($ Eff. Born v0 $)-A_{F B}($ Improved Born, with boxes $)$ & -0.00039 & -0.00042 \\
$A_{F B}\left(\right.$ Eff. Born v1) $-A_{F B}($ Improved Born, with boxes $)$ & -0.00042 & -0.00042 \\
$A_{F B}($ Eff. Born v2 $)-A_{F B}($ Improved Born, with boxes $)$ & -0.00022 & -0.00024 \\
\hline
\end{tabular}

DIZET 6.45 form factors and running width were used in re-weighting of LHC $p p \rightarrow Z j ; Z \rightarrow l^{+} l^{-}$ events simulated at $8 \mathrm{TeV}$. From the first two lines, magnitude of EW corrections with respect to lowest order, $\alpha(0)$ scheme. Following three lines demonstrate precision of Effective Born variants with respect to Improved Born

function of energy, scattering angle and flavour of incoming partons (it can be an electronpositron pair as well). The integrated over angle partonic cross section $\sigma^{\text {tot }}, A_{F B}$ and $P_{\tau}$ can be drawn. Comparison plots can be prepared, either with the help of the FFdrawDwa. C script to compare results with EW form factors obtained with variants of DIZET initialization, or with FFdraw. C to compare Improved Born and Effective Born of the choice as implemented in TAUOLA package. For technical details see Appendix C. An example results for comparison of Effective Born as encapsulated in TauSpinner/Tauola (version of December 2019) defaults and Improved Born with EW form factors of DIZET 6.45 are shown in Fig. 3. One should note that differences between Improved Born and Effective Born, even in case when detailed tuning of parameters is not performed, are not large from the perspective of many applications.

Numerical results, as in previous section, are monitored with $\sin ^{2} \theta_{W}^{e f f}, P_{\tau}, A_{F B}$ and partonlevel $\sigma^{\text {tot }}$. In Fig. 8 and Table 4, it is shown how results depend on the library version. The presentation in Table 4 includes predictions on $\alpha\left(M_{Z}^{2}\right), M_{W}, \Delta r$ and $\sin ^{2} \theta_{W}^{\text {eff }}$. Further results are delegated to Appendices.

By inspection of Table 4, one can conclude that the choice of EW library variant is not of great importance, unless precision better than $20 \cdot 10^{-5}$ on $\sin ^{2} \theta_{W}^{\text {eff }}$ is required. Even if precision requirements are not very demanding one should keep in mind that below $40 \mathrm{GeV}$ in older versions of DIZET the hadronic part of $\Pi_{\gamma \gamma}(s)$ was set to zero. See also Fig. 3 for minor discontinuity at $30 \mathrm{GeV}$ due to edge of tabulation zones. Further details on the impact of change of options/flags of DIZET 6.45 are collected in Tables 8 and 9 of Appendix B.2.

\subsection{Parametric uncertainties}

The precision of the EW calculations depends not only on the EW scheme used for the calculations (see, for example, [38,39], but also on imposed set of input parameters and corresponding parametric uncertainties. Parametric uncertainties are defined as the ones due to uncertainties of EW calculation inputs, such as $m_{t}, M_{W}$ or $\Delta \alpha_{h}^{(5)}(s)$. That is the reason why precision of these input parameters (taken from measurements), specially $M_{W}$ or $\sigma_{e+e-\rightarrow \text { hadrons }}^{\text {tot }}(s)$ (for $\alpha_{Q E D}\left(M_{Z}^{2}\right)$ ), is of importance. For clarity of the presentation, this topic is covered only in Appendices B.2, B.3, in particular in Tables 8, 9, 10, and 11. We show how some phenomenologically sound quantities depend on initialization uncertainties for $\alpha(0)$ EW scheme used in the DIZET library. In particular how do they depend on: (i) distinct $\Delta \alpha_{h}^{(5)}(s)$ parametrization, (ii) uncertainty from changing $\Delta \alpha_{h}^{(5)}\left(M_{Z}^{2}\right)$ by \pm 0.0001 , (iii) 

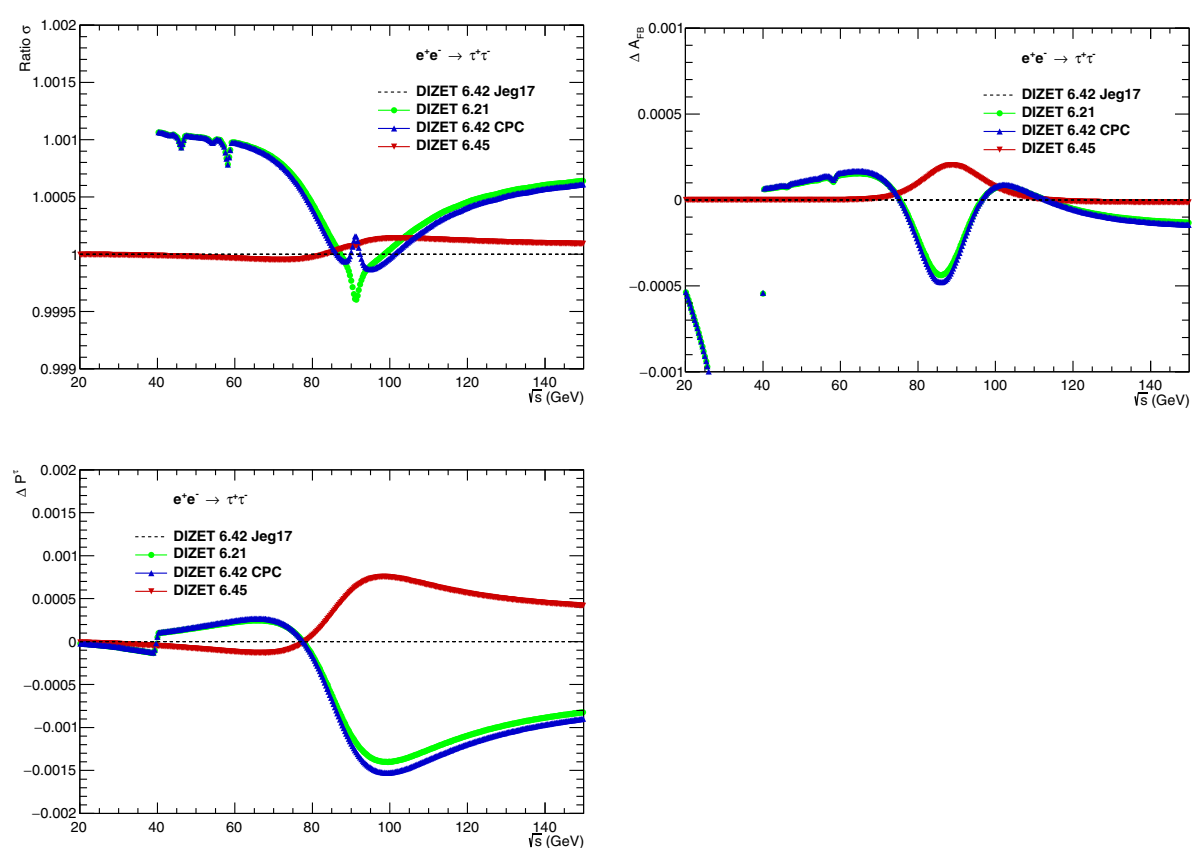

Fig. 8 Comparison of $\sigma_{t o t}\left(m_{\tau^{+} \tau^{-}}\right), A_{F B}\left(m_{\tau^{+} \tau^{-}}\right)$and $P_{\tau}\left(m_{\tau^{+} \tau^{-}}\right)$, obtained from TauSpinner calculations of Improved Born and EW tables calculated with the DIZET libraries. As a reference, version 6.42 improved with photon vacuum polarization of Ref. [99] is used. Note that interface of photon vacuum polarization of DIZET 6.42 and 6.21 prevented its calculation below $40 \mathrm{GeV}$. This, and other minor parameter variation in particular of $M_{Z}$ and $\Gamma_{Z}$, lead to bumps on the plots which do not need to be investigated now, and even for the precision tests of the SM at LHC, as they are smaller than precision requirements. On the other hand, proper adjustment for the effects important at (and around) the $Z$ peak need to be performed. The corresponding EW-corrected $M_{W}, \sin ^{2} \theta_{W}^{e f f}$ and $\alpha$ at the $Z$ pole are collected in Table 4. Similar results can be obtained from Tauspinner for the quark-level Effective Born and Improved Born predictions

uncertainty due to top mass change by $\pm 0.5 \mathrm{GeV}$. The estimated total parametric uncertainty for EW $\alpha(0)$ scheme used for predicting $\sin ^{2} \theta_{W}^{e f f}\left(M_{Z}^{2}\right)$ is about 0.00005 .

To summarize, these example results can be useful by themselves. They can also help for the choice of the EW renormalization and input schemes. The particular choice of scheme, even if uncertainty due to missing higher-order terms is small, may turn out non-optimal, if its input parameters introduce into predictions sizable parametric uncertainties.

\section{Summary}

One loop EW corrections play an important role in the precision tests of the Standard Model. At the same time, other effects related to special classes of higher-order corrections had to be taken into account. That is the reason, why special libraries of EW corrections were developed, maintained and gradually improved. Over the last 30 years, the DIZET library was established as a prominent one. In principle, DIZET relies on one-loop calculations, but it is supplemented with dominant higher-order terms. It is sometimes called as an approach at 1.5 loop level. Special role is played by the so-called EW $\alpha(0)$ scheme (with $G_{\mu}, \alpha(0)$, $M_{Z}$ input). This scheme and DIZET library found large spectrum of applications, not only 
Table 4 Predictions for different versions of DIZET 6. explained in Appendix B

\begin{tabular}{lcccc}
\hline Parameter & DIZET 6.21 & $\begin{array}{l}\text { DIZET 6.42 } \\
\text { CPC }\end{array}$ & $\begin{array}{l}\text { DIZET 6.42 } \\
\text { (Jeg. 2017) }\end{array}$ & DIZET 6.45 \\
\hline$\alpha\left(M_{Z}^{2}\right)$ & 0.007759954 & 0.007759954 & 0.0077549256 & 0.0077549256 \\
$1 / \alpha\left(M_{Z}^{2}\right)$ & 128.86674175 & 128.86674175 & 128.95030206 & 128.950302056 \\
$M_{W}(\mathrm{GeV})$ & 80.3560012 & 80.3535973 & 80.3621285 & 80.3589358 \\
$\Delta r$ & 0.03676619 & 0.03690875 & 0.03640232 & 0.03633354 \\
$\Delta r_{\text {rem }}$ & 0.01168031 & 0.01168001 & 0.01168106 & 0.01168393 \\
$s_{W}^{2}$ & 0.22345780 & 0.22350426 & 0.22333937 & 0.22340108 \\
$\sin ^{2} \theta_{W}^{\text {eff lepton }}\left(M_{Z}^{2}\right)$ & 0.23173519 & 0.23174233 & 0.23157947 & 0.23149900 \\
$\sin ^{2} \theta_{W}^{\text {eff up-quark }}\left(M_{Z}^{2}\right)$ & 0.23162861 & 0.23174233 & 0.23147298 & 0.23139248 \\
$\sin ^{2} \theta_{W}^{\text {eff down-quark }}\left(M_{Z}^{2}\right)$ & 0.23150149 & 0.23174233 & 0.23134599 & 0.23126543 \\
\hline
\end{tabular}

The $\Delta r, \Delta r_{e m}$ represent corrections to $M_{W}$ calculations, see Eq. (8), where $s_{W}^{2}=1-\frac{M_{W}^{2}}{M_{Z}^{2}}$

in phenomenology of LEP $e^{+} e^{-}$collisions but $p \bar{p}$ and $p p$ Tevatron and LHC experiments as well. The choice of the scheme was motivated to minimize parametric uncertainties, which are larger if $\alpha\left(M_{Z}\right)$ or $M_{W}$ is used as input as in case of some other schemes. An attempt to archive distinct versions of EW libraries was made in [26]. These results and methods may need to be reproducible at the time of future FCC or other future experiments. Further advantages of $\alpha(0)$ scheme are that when projected to Effective Born, the bulk of EW corrections is embedded into effective couplings. Also, QED effects or strong interaction effects of initial state can be separated out, and thus, it is possible to treat them in distinct calculation schemes and in particular to higher order.

From our investigations, see Sects. 4.1, and 4.2, we can conclude that effective couplings approach (Effective Born) remains sufficient for predictions, where precision requirements do not exceed $\sim 20 \cdot 10^{-5}$ for $\sin ^{2} \theta_{W}^{e f f}$. Re-scaling of coupling constants, $Z$ boson width may be still sufficient. That observation may simplify the phenomenology challenges for LHC observations, where precision at present is at the level of $30 \cdot 10^{-5}$. Effective Born versions $v 1$ or $v 2$ may be used with strong interaction studies: fits of PDF, higher-order QCD calculations, etc. Note that main challenges for precision of electroweak measurements are localized in strong interactions. Beyond $\sim 20 \cdot 10^{-5}$ precision level, use of Improved Born without simplifications is needed, and TauSpinner can be used for its implementation. However, once precision requirements become sizably better than $20 \cdot 10^{-5}$, this may turn out not to be sufficient, as it can be concluded from observations at ends of Sects. 4.1, 4.2 and also from Table 4, the last three lines. Even in the FCC-ee case, when requirements for the precision are particularly strict, $\sin ^{2} \theta_{W}^{e f f}$ can be used to enumerate which effects must be taken into account in calculations and in production of the numerical results.

Presented implementation of TauSpinner weights enable discussion of particular classes of higher-order EW effects, without need to recalculate corrections of strong interactions. We have installed algorithms useful for that purpose into Tauspinner library. Examples of numerical results are focused on centre-of-mass system energy dependence of total cross sections, forward-backward asymmetry of leptons and $\tau$ lepton polarization in $e^{+} e^{-}$and $p p$ collisions. 
New TauSpinner algorithms have potential to improve EW effects in simulation samples obtained from programs predominantly of strong interactions. We have shown results of re-weighting with different levels of sophistication for implementation of EW corrections. In the present paper, we have explained how the numerical impact of some effects of EW results can be imprinted into broad spectrum of simulation samples where EW loop effects are missing, or impact of their initialization is to be studied.

Finally, let us point that in future, for higher-order high-precision EW calculations, TauSpinner/DIZET algorithms still may be useful for evaluation which contributions (and to which order) need to be taken into account to attain requested precision level. Some details, considered negligible once, may turn out important for the comparisons with future measurements. Even if such high precision cannot be achieved with solutions envisaged in the paper, they may still suffice to evaluate what classes of corrections need to be included in future calculations.

Acknowledgements E.R-W. would like to thank Daniel Froidevaux and colleagues from ATLAS Collaboration Standard Model Working Group for numerous inspiring discussion on the applications of presented here implementation of EW corrections to the $\sin ^{2} \theta_{W}^{e f f}$ measurement at LHC. This project was supported in part from funds of Polish National Science Centre under decisions DEC-2017/27/B/ST2/01391 and partly supported by the CERN FCC Design Study Program. Majority of the numerical calculations were performed at the PLGrid Infrastructure of the Academic Computer Centre CYFRONET AGH in Krakow, Poland.

Open Access This article is licensed under a Creative Commons Attribution 4.0 International License, which permits use, sharing, adaptation, distribution and reproduction in any medium or format, as long as you give appropriate credit to the original author(s) and the source, provide a link to the Creative Commons licence, and indicate if changes were made. The images or other third party material in this article are included in the article's Creative Commons licence, unless indicated otherwise in a credit line to the material. If material is not included in the article's Creative Commons licence and your intended use is not permitted by statutory regulation or exceeds the permitted use, you will need to obtain permission directly from the copyright holder. To view a copy of this licence, visit http://creativecommons.org/licenses/by/4.0/.

\section{A Electroweak phenomenology in nut-shell}

Electroweak corrections represent a complex subject and it is challenging to separate it from the other parts of physics processes calculations [2]. In general, neither Effective Born nor Improved Born can be used directly, but corresponding amplitudes are used as building blocks of complete scattering amplitudes. For $e^{+} e^{-}$collisions, sizable fraction of events represent configurations with hard bremsstrahlung photons and for $p p$ collisions events of DrellYan lepton pairs are usually accompanied with hadronic jets. It is thus easy to realize that higher-order, real and loop corrections are indispensable for realistic predictions matching experiments' precision. This is challenging effort, which was avoided in the past, and in some cases will be hopefully avoided in future as well. At present, one can already for many cases rely on NLO QCD+EW calculations [40]. QED and strong interaction effects require higher-order predictions but not necessarily higher than one-loop genuine weak calculations. In particular, infrared singularities were left outside of weak calculations. Also, in some circumstances dominant loop corrections were identified as gauge invariant groups, and thus iterated to include dominant higher-order corrections.

The initial state corrections are those which are proportional to square of incoming to the hard process electrons (or quarks) charge. The corresponding real emission amplitudes are also proportional to the same charge. Final state corrections are proportional to the square of outgoing partons charge. They separate and are individually gauge invariant, because of 
distinct coefficients of charge. The interference of the two contributions and corresponding box are proportional to the product of initial and final state charges, thus also of distinct coefficient from the previous ones; must be thus individually gauge invariant. Box diagrams of two boson lines both attached to incoming and outgoing fermion lines contribute corrections which are scattering angle dependent. Finally, the vacuum polarization is the loop corrections attached on both sides to the propagator of interaction boson. Analytical form of such contribution does not depend on the fermion flavour loop, which may differ from the one of incoming or outgoing fermion. Thus are gauge invariant as well. This correct, but simplistic set of arguments and definitions applies to the case of fermion anti-fermion pair production from annihilation of incoming pair of fermions of different flavours. If $t$-channel exchange contributes at the lowest order, or higher-order corrections are taken into account, picture becomes more complicated, but still does not need to be abandoned in full. Gauge invariant parts may be identified with the help of other processes. For example, amplitude for $e^{+} e^{-} \rightarrow v_{\mu} \bar{v}_{\mu}$ constitutes gauge invariant part for $e^{+} e^{-} \rightarrow v_{e} \bar{v}_{e}$ one, but once electroweak bosons and interactions are taken into account picture complicates further.

It is of no surprise that discussing this subject is far beyond the scope of the present publication. Nonetheless, some, even if incomplete, review of results and concepts used is given. Reader interested in foundations of the schemes may find wealth of important information from the monumental LEP-time book on the subject [41]. A recent Report [13] on EW effects and in particular on matching these effects with strong interactions cover many solutions developed over the last two decades.

\section{A.1 Topic establishing}

From the beginning, already at LEP 1, the subject required complex approaches $[42,43]$. Some of the programs developed at that time are still used today [9]; other became less popular, even if in their development important results were obtained $[44,45]$ and found application in broader domain [46]. Let us indicate few important paths which were taken, as it may be helpful to evaluate usefulness and application limits, of results presented here.

Already at LEP 2 [47] some of the calculation schemes [2] used at LEP 1 became painfully difficult, because at higher energies EW boson exchanges in $t$-channel had to be taken into account, and thus, gauge dependence cancellation between diagrams of $s$ - and $t$-channel boson exchanges had to be assured. However, as precision requirements were less of an issue, this could have been resolved simply by neglecting EW loop corrections and form factors breaking the cancellations. This of course is not anymore the case at present and will become even more difficult in the future, with increasing precision measurements and reactions energies, where multi-boson final states will contribute significantly. Let us point to Ref. [48], for example, of forth-going efforts in that direction. We will limit our attention to hard scattering processes of virtualities and transverse momenta, not exceeding significantly the $Z$ boson mass. But possibly some of our observations may be indirectly useful for that energy regions as well.

In the following, let us turn to the fundamental point how Electroweak corrections for $2 \rightarrow 2$ processes and of the one loop level can be used together with the dominant higherorder effects and for kinematic configurations, with additional partons or photons in the final states. 
A.2 The $Z$ boson width and $Z, \gamma$ propagators

Obviously, the first is the question of how to deal with electroweak boson width, which is formally higher-order correction itself. From the very beginning, analytic continuation of boson mass to complex value was used. A formal and sophisticated approach [49] was published much later [44]. The approach [46] relies on this work. There, it is underlined that analytic continuation to complex masses requires to be supplemented with careful study of consequences for the unitarity, in particular for Cutkosky rules [12]. To this end, special approach to the perturbation expansion was designed and in particular, boson lines, which may be cut, are treated differently for amplitude and its complex conjugate. Introduction of 1 to 1 vertex (with the coupling corresponding to boson width) was helpful, and $Z$ boson propagator necessary for resonance description was established [46].

Nearly immediately it turned out that fixed width in propagator was not sufficient for numerical predictions. On the other hand, extensions were considered to be conceptually challenging, and, for example, in ZOPOLE program [45] developed simultaneously with work on Ref. [44], even dominant gluon exchange corrections to $Z$ boson vacuum polarization were not included at the beginning. That is why this otherwise beautiful program, interfaced and archived with KORALZ Monte Carlo [50] was never used for phenomenological purposes.

Practical solution was that whenever diagrams of fermion loop, i.e. bosons vacuum polarizations $\Pi_{\gamma \gamma}, \Pi_{\gamma Z}, \Pi_{Z Z}$ could be encapsulated in gauge invariant part, higher order effects could be added. In fact, it turned out that even third-order strong interaction corrections were necessary to be included in calculations aiming at comparisons with the data $[9,11]$. Also, non-perturbative $\Pi_{\gamma \gamma}$ contributions could be included, thanks to dispersion relations and measured low energy $e^{+} e^{-} \rightarrow$ hadrons total cross section data. The same reasoning enables that in many applications, so-called running $Z$ width was used and sufficient to encapsulate dominant parts of the higher-order corrections to $Z$ boson propagator. Note that this implies including some, but not all contributions of higher order, which may be of concern.

$$
\alpha_{Q E D}\left(M_{Z}^{2}\right)
$$

One may point to a related aspect of precision calculation, that is calculation scheme. At present, $\alpha_{Q E D}(0)$ is used among input in so-called EW $\alpha(0)$ scheme (defined by $G_{\mu}, \alpha(0)$ and $\left.M_{Z}\right)$. This is the reason why $\alpha_{Q E D}\left(M_{Z}^{2}\right)$ needs to be obtained with the help of dispersion relations and low energy data. This inevitably leads to uncertainties.

If $\alpha_{Q E D}\left(M_{Z}^{2}\right)$ could be measured directly that would help to reduce parametric uncertainty due to the choice of input parameter scheme [38] (Section 3.4.3), it may be the case at FCC [4] (page 5 Table A.1.2). However, at present, such parametric uncertainties especially of $M_{W}$ mass measurement are of importance and need to be taken into account in choice of the most optimal calculation schemes.

\section{A.3 Effective/Improved Born and bremsstrahlung or jets}

Matching with higher-order strong and QED effects which affect final states composition cannot be ignored. Already at the beginning of LEP 1 it was realized that initial state QED bremsstrahlung reduces cross section at the $Z$ peak, by about $40 \%$. For that, higher-order corrections were necessary [51-53] and could be included thanks to not only higher-order QED fixed-order calculation, but exponentiation technique used as well. Similar or even larger in size strong interaction corrections need to be taken into account at hadron colliders. 
For some processes, genuine weak sector may be treated separately from jet (or QED hard bremsstrahlung) matrix elements. This may be the case of FCC-hh physics, and then, solutions like implemented in TauSpinner may be helpful.

Let us focus on the example, where gauge invariance and other techniques were used to order the calculations. This is the case where QED effects were separated from complete electroweak ones, so they could be taken to higher orders.

\section{A.4 Separating-matching weak and QED corrections}

In KKMC Monte Carlo of $e^{+} e^{-} \rightarrow l^{+} l^{-} n \gamma$ processes, where second-order QED matrix element and coherent exclusive exponentiation is used [10,24], spinor techniques of KleissStirling [54] are used for matrix element calculations [55]. If electroweak form factors were introduced to spin amplitudes of $e^{+} e^{-} \rightarrow l^{+} l^{-} \gamma \gamma$, non-physical huge contributions, dramatically larger than any logarithmic one, proportional to $s / m_{e}^{2} \simeq 10^{10}$ would appear. However, this is not the case as contributions to Yennie-Frautchi-Suura spin amplitude level $\beta_{0}, \beta_{1}, \ldots$ terms are calculated, and gauge cancellations are explicitly performed, infrared singular factors localized, before EW form-factor installation. That was particularly demanding in case of $e^{+} e^{-} \rightarrow v_{e} \bar{v}_{e} \gamma \gamma$ production, because $t$-channel $W$ boson exchange complicated cancellations of gauge parameter dependence, see [56] for the $e^{+} e^{-} \rightarrow v \bar{v} \gamma$ and for discussion of $e^{+} e^{-} \rightarrow v_{e} \bar{v}_{e} \gamma \gamma$ Ref. [57].

\section{A.5 Residual angular dependencies}

Even in this studied in detail KKMC case, one needs to evaluate if cancellation of real emissions and virtual corrections contributions to the initial-final state QED interference is not damaged. This cancellation may be obscured by energy angular dependence of form factors. Formally of higher order, such mismatches could impact some predictions if attention is not paid. It needs to be checked if results like of Refs. [58,59] on QED initial-final state interference, which were important at the time of program design, still hold at new higher precision regime [60].

At LEP 1, dominated measurements at energies are close to the $Z$ peak. There are two reasons why then angular dependence of EW corrections embedded in form factors is reduced and description of Effective Born was used. The Effective Born, as discussed in Sect. 3.1, means re-defining coupling constants to the values incorporating dominant contributions from the higher-order (loop) corrections (at fixed energy, e.g. $\sqrt{s}$ or $M_{Z}$ ). At energies below $W W$ channel opening, that is below $W$ pair production threshold the EW boxes cannot contribute in a double resonant way, see, for example, [9], effect is numerically small. That is the same reason why so-called EW Sudakov form factors become numerically important at higher energies only. It is well known, for the review, see [47], the properties are used in DIZET library [41].

Another reason is of a more physical nature. The following heuristic argument can be used. Lifetime of the $Z$ boson even if small separates phenomena of incoming beams and outgoing leptons, that is why angular dependence of amplitudes at energies close to $Z$ peak reduces to the one of Born-level point-like interactions; that is defined only by spin $1 / 2$ of incoming and outgoing leptons and 1 for intermediate $Z / \gamma$. Simply, angular-dependent terms of virtual and real emission corrections do not become enlarged in proportion to the $Z$ peak and thus appear relatively smaller than off the peak. Similarly, QED initial-final state interference is reduced by the lifetime of the $Z$ boson too [58]. 
For the presented above reasoning, QED part need to be carefully separated out in fully differential form [10]. We follow Refs. $[9,61]$ for definitions of line shape corrections $\left[\Gamma_{V_{\Pi}}\right.$ and $Z_{V_{\Pi}}$ of Eq. (1)] and pole (double pole) approximation. Finally, the remaining parts of EW corrections are called genuine weak corrections and are defined at spin amplitude level.

A.6 EW-sensitive observables and $\sin ^{2} \theta_{W}^{\text {eff }}$

At higher energies and higher luminosities and already at LHC, number of processes where electroweak effects need to be taken into account is much larger, even if not in all cases the same level of precision needs to be assured. Often complexity of corresponding QCD calculation largely surpass this of electroweak ones. That is why, it is of importance to evaluate, when and to which precision electroweak corrections can be treated separately and if necessary, which simplifications can be introduced enabling calculations for initial and final states interactions, without loss of an overall precision. To evaluate sensitivity to electroweak parameters and precision requirements, for any observable, used already at LEP 1 time or introduced later, uncertainty is translated to the one of $\sin ^{2} \theta_{W}^{e f f}$, which is then used as an universal measure.

Whether it can be used directly, or if it can be used to enumerate bulk of the effects, with some corrections still needed, $\sin ^{2} \theta_{W}^{e f f}$ remains useful as pseudo-observable.

It became a standard for comparisons of observables not only of LEP 1 [2], but of later measurements at Tevatron [62] and LHC [5,6] as well. It is used in investigations of physics potential for future measurements at HL-LHC [7,8] and FCC [4] as well. For that to be useful, one needs to investigate its suitability in new conditions, at HL-LHC and at FCC of much higher precision than at LEP 1. In these cases, even at lowest approximation level several parton-level Born amplitudes contribute simultaneously for each individual event.

For numerical predictions of higher precision, Effective Born with couplings defined by $\sin ^{2} \theta_{W}^{e f f}$ may be insufficient to emulate complete electroweak corrections. Introduction of flavour dependent variants of $\sin ^{2} \theta_{W}^{e f f}$ may help [2], but not in every case though.

Effective Born predictions for the particular observable are easy to vary with $\sin ^{2} \theta_{W}^{e f f}$ shifts of its uncertainty range or due to some particular contribution. It may be helpful to evaluate what is necessary for the required precision, even if complete electroweak corrections are at the end found to be necessary. That is also why, it is of importance to investigate where are the limits of feasibility for pictures of Improved and Effective Born approximations, the pictures which largely simplify complexity of QCD calculations for $p p, p \bar{p}$ collisions, or QED for $e^{+} e^{-}$.

\section{A.7 Outlook}

Problems for merging electroweak and QCD effects are inevitably more complicated than in discussed above QED case predominantly of $s$-channel $Z / \gamma^{*}$ exchange only. That is why, in programs such as [63-66] electroweak loop corrections are usually not included, for the example discussion on matching QCD and EW corrections, see Ref. [67]. It is even envisaged [68] that not only electroweak form factors cannot be used, but in some cases constant width in $Z$ propagator as of Eq. (12) has to be used; the running one of Eq. (11) avoided. Running $Z$ width represent approximation of some loop contributions [69], an approximation valid at relatively lower energies, but not universally.

That may lead to unacceptable for experimental data analysis precision loss, either because of approximation in electroweak or in strong interaction sector. As QCD effects and uncertain- 
ties are larger, one may ask which, facilitating tasks for QCD, simplifications in electroweak sector can be acceptable from numerical perspective.

In general, one want to use results of Quantum Field Theory, rather than inspired by it, but otherwise ad hoc models. Theoretical conditions need to be respected. In many cases solutions of LEP times, as presented and adopted in the main part of the paper, may be helpful. But this is not granted, every case need to be investigated separately and again when precision requirements improve.

\section{B DIZET EW corrections}

The DIZET package relies on on-mass-shell (OMS) renormalization scheme [70,71], and the $\left(G_{\mu}, \alpha(0), M_{Z}\right)$ are the principal input parameters, dependence on $m_{h}$, top quark and lepton masses are numerically less important. The OMS input scheme includes masses of all fundamental particles, fermions $Z$ and $h$ bosons, electromagnetic coupling constant $\alpha(0)$ and strong coupling $\alpha_{s}\left(M_{Z}\right)$. The OMS is used with modifications. The dependence on the ill-defined masses of the light quarks $u, d, c, s$ and $b$ is solved by dispersion relation, for details see [9]. Another exception is $W$-boson mass $M_{W}$, which still can be predicted with better theoretical uncertainties than experimentally measured value, exploiting the very precise knowledge of the Fermi constant in $\mu$-decay $G_{\mu}$. The discussed above EW scheme is in the literature often called EW $\alpha(0)$ scheme [2]. The $M_{W}$ is calculated iteratively from the equation

$$
M_{W}=\frac{M_{Z}}{\sqrt{2}} \sqrt{1+\sqrt{1-\frac{4 A_{0}^{2}}{M_{Z}^{2}(1-\Delta r)}}},
$$

where

$$
A_{0}=\sqrt{\frac{\pi \alpha(0)}{\sqrt{2} G_{\mu}}} .
$$

The Sirlin's parameter $\Delta r$ [72]

$$
\Delta r=\Delta \alpha\left(M_{Z}\right)+\Delta r_{L}+\Delta r_{e m}
$$

is also calculated iteratively, and the definition of $\Delta r_{L}, \Delta r_{e m}$ involves re-summation of vacuum polarization loop corrections and also some higher-order corrections. Since this term implicitly depends on $M_{W}$ and $M_{Z}$, iterative procedure is needed. The re-summation contribution in formula (8) is not formally justified by renormalization group arguments, and correct generalization is to compute higher-order corrections, see more discussion in [9]. The electromagnetic coupling evolves from Thomson limit and for $Z$-boson energy scale receives corrections

$$
\Delta \alpha\left(M_{Z}\right)=\Delta \alpha_{h}^{(5)}\left(M_{Z}\right)+\Delta \alpha_{l}\left(M_{Z}\right)+\Delta \alpha_{t}\left(M_{Z}\right)+\Delta \alpha^{\alpha \alpha_{s}}\left(M_{Z}\right)
$$

The hadronic vacuum polarization correction is contained in the quantity denoted as $\Delta \alpha_{h}^{(5)}\left(M_{Z}\right)$, which is treated as one of the input parameters. It can be either computed from quark masses or, preferably, fitted to experimental low energy $e^{+} e^{-} \rightarrow$ hadrons data [73]. The leptonic loop correction $\Delta \alpha_{l}\left(M_{Z}\right)$ is calculated analytically. Both $\Delta \alpha_{h}^{(5)}\left(M_{Z}\right)$ and $\Delta \alpha_{l}\left(M_{Z}\right)$ are significant, respectively, about 0.0275762 and 0.0314976 , and the remaining terms are rather marginal, respectively, about $-5 \cdot 10^{-5}$ and $-1 \cdot 10^{-5}$. 
In the OMS renormalization scheme, the weak mixing angle is defined uniquely through the gauge-boson masses:

$$
\sin ^{2} \theta_{W}=s_{W}^{2}=1-\frac{M_{W}^{2}}{M_{Z}^{2}} .
$$

With this scheme, measuring $\sin ^{2} \theta_{W}$ would be equivalent to indirect measurement of $M_{W}^{2}$ through the relation (10).

\section{B.1 Initialization flags and input parameters}

The recommended sets of flags are quite stable since 1995, and new options consider updated parametrizations of the vacuum polarization hadronic corrections $\Delta \alpha_{\text {had }}^{(5)}$ (flag IHVP), updated calculations for two loop fermionic corrections (flag IAMT4) and updated three-loop corrections (flag IAFMT).

In Table 5, we collected information on the initialization flags recommended for different versions of DIZET $6 . \mathrm{XX}$. For detailed information about meaning of the individual flags, see DIZET $6 . X X$ documentations $[9,25,74]$.

Let us here just explain those, for which recommended values have changed since version DIZET 6.21:

- Switch for Hadronic vacuum polarization corrections $\Delta \alpha_{\text {had }}^{(5)}$ :

IHVP $=1$ parametrization of [75]

IHVP $=5$ parametrization of [73]

- Switch for re-summation of the leading $O\left(G_{f} m_{t}^{2}\right)$ EW corrections:

IAMT $4=4$ with two-loop sub-leading corrections and re-summation [76-79]

IAMT $4=5$ with fermionic two-loop corrections to $M_{W}$ [80-82]

IAMT $4=6$ with complete two-loop corrections to $M_{W}[83]$ and fermionic $\sin ^{2} \theta_{W}^{\text {eff lep }}$ two-loop corrections [84]

IAMT $4=7$ with complete two-loop corrections to $\sin ^{2} \theta_{W}^{\text {eff lep }}$ and $\sin ^{2} \theta_{W}^{e f f l b}[85,86]$

IAMT $4=8$ with complete two-loop corrections to $\sin ^{2} \theta_{W}^{\text {eff }}$ [87]

- Switch for three-loop corrections $O\left(\alpha \alpha_{s}^{2}\right)$ to the EW $\rho$ parameter:

IAFMT $=1$ corrections $O\left(G_{\mu} m_{t}^{2} \alpha_{s}^{2}\right)$ included [88]

IAFMT $=2$ corrections $O\left(G_{\mu} m_{t}^{2} \alpha_{s}^{2}\right), O\left(G_{\mu} M_{Z}^{2} \alpha_{s}^{2}+\log \left(m_{t}^{2}\right)\right)$ included IAFMT $=3$ corrections $O\left(G_{\mu} m_{t}^{2} \alpha_{s}^{2}\right), O\left(G_{\mu} M_{Z}^{2} \alpha_{s}^{2}+\log \left(m_{t}^{2}\right)\right)$ and $O\left(G_{\mu} M_{Z}^{2} / m_{t}^{2} \alpha_{s}^{2}\right)$ included

Since LEP time physics measurements evolved, and as a consequence initialization parameters as well. For the recent status summary see last edition by Particle Data Group [89]. The Higgs boson has been discovered at LHC and its mass measured with precision of $25 \mathrm{MeV}$ [90]. The $\mathrm{W}$ boson mass in known at LHC with precision better than $18 \mathrm{MeV}$ [91] and the top mass is known with precision much better than $1 \mathrm{GeV}$ [92].

In Tables 6 and 7, we collected initialization parameters: masses and couplings, used of the paper numerical evaluation. The exact values of some of them, which serve as benchmark values for different comparisons, have been chosen as such to be fully compatible with the ongoing studies of the LHC EW Working Group [93].

\section{B.2 Numerical results}

The DIZET library, when invoked, provides tabulated $s, t$-dependent form factors. It calculates also $M_{W}$, Stirling parameter $\Delta r, \Delta r_{e m}$ and flavour-dependent $\sin ^{2} \theta_{W}^{e f f}$ at $Z$ peak. In 
Table 5 DIZET initialization flags: different versions defaults

\begin{tabular}{|c|c|c|c|c|c|}
\hline Input NPAR() & Internal flag & $\begin{array}{l}\text { DIZET } 6.21 \\
\text { Defaults in [25] }\end{array}$ & $\begin{array}{l}\text { DIZET } 6.42 \\
\text { Defaults in [74] }\end{array}$ & DIZET 6.45 & Comments \\
\hline NPAR(1) & IHVP & 1 & 1 & 5 & $\begin{array}{l}\Delta \alpha_{\text {had }}^{(5)} \text { param. } \\
\text { from [73] in } \\
\text { v6.45 }\end{array}$ \\
\hline NPAR(2) & IAMT4 & 4 & 4 & 8 & $\begin{array}{l}\text { New development } \\
\text { in v6.42, v6.45 }\end{array}$ \\
\hline NPAR(3) & IQCD & 3 & 3 & 3 & \\
\hline NPAR(4) & IMOMS & 1 & 1 & 1 & $\begin{array}{l}M_{W} \text { calculated } \\
\text { with formula (6) }\end{array}$ \\
\hline NPAR(5) & IMASS & 0 & 0 & 0 & \\
\hline NPAR(6) & ISCRE & 0 & 0 & 0 & \\
\hline NPAR(7) & IALEM & 3 & 3 & 3 & \\
\hline NPAR(8) & IMASK & 0 & 0 & 0 & $\begin{array}{l}\text { Not used since } \\
\text { v6.21 }\end{array}$ \\
\hline NPAR(9) & ISCAL & 0 & 0 & 0 & \\
\hline NPAR(10) & IBARB & 2 & 2 & 2 & \\
\hline NPAR(11) & IFTJR & 1 & 1 & 1 & \\
\hline NPAR(12) & IFACR & 0 & 0 & 0 & \\
\hline NPAR(13) & IFACT & 0 & 0 & 0 & \\
\hline NPAR(14) & IHIGS & 0 & 0 & 0 & \\
\hline NPAR(15) & IAMFT & 1 & 3 & 3 & \\
\hline NPAR(16) & IEWLC & 1 & 1 & 1 & \\
\hline NPAR(17) & ICZAK & 1 & 1 & 1 & \\
\hline NPAR(18) & IHIG2 & 1 & 1 & 1 & \\
\hline NPAR(19) & IALE2 & 3 & 3 & 3 & \\
\hline NPAR(20) & IGREF & 2 & 2 & 2 & \\
\hline NPAR(21) & IDDZZ & 1 & 1 & 1 & \\
\hline NPAR(22) & IAMW2 & 0 & 0 & 0 & \\
\hline NPAR(23) & ISFSR & 1 & 1 & 1 & \\
\hline NPAR(24) & IDMWW & 0 & 0 & 0 & \\
\hline NPAR(25) & IDSWW & 0 & 0 & 0 & \\
\hline
\end{tabular}

Table 6 The EW parameters used at tree-level EW, with on-mass-shell definition (LEP convention)

\begin{tabular}{ll}
\hline Parameter & $\left(\alpha(0), G_{\mu}, M_{Z}\right)$ \\
\hline$M_{Z}(\mathrm{GeV})$ & 91.1876 \\
$\Gamma_{Z}(\mathrm{GeV})$ & 2.4952 \\
$\Gamma_{W}(\mathrm{GeV})$ & 2.085 \\
$1 / \alpha$ & 137.035999139 \\
$\alpha$ & 0.007297353 \\
$G_{\mu}\left(\mathrm{GeV}^{-2}\right)$ & $1.1663787 \cdot 10^{-5}$ \\
$M_{W}(\mathrm{GeV})$ & 80.93886 \\
$s_{W}^{2}$ & 0.2121517 \\
$\alpha_{S}\left(M_{Z}\right)$ & 0.12017890 \\
\hline
\end{tabular}


Table 7 Values of fermions and Higgs boson masses used for calculating EW corrections

\begin{tabular}{lll}
\hline Parameter & Mass $(\mathrm{GeV})$ & Description \\
\hline$m_{e}$ & $5.1099907 \mathrm{e}-4$ & Mass of electron \\
$m_{\mu}$ & 0.1056583 & Mass of muon \\
$m_{\tau}$ & 1.7770500 & Mass of tau \\
$m_{u}$ & 0.0620000 & Mass of up-quark \\
$m_{d}$ & 0.0830000 & Mass of down-quark \\
$m_{c}$ & 1.5000000 & Mass of charm-quark \\
$m_{s}$ & 0.2150000 & Mass of strange-quark \\
$m_{b}$ & 4.7000000 & Mass of bottom-quark \\
$m_{t}$ & 173.0 & Mass of top quark \\
$m_{H}$ & 125.0 & Mass of Higgs boson
\end{tabular}

Table 4, we have collected numerical results on predicted masses and couplings, including EW corrections. Those values come directly as control printout from DIZET 6 . XX code. In total, evolution of the implemented EW corrections leads to shift in the predicted $M_{W}$ by $+3 \mathrm{MeV}$, on-shell $s_{W}^{2}$ by -0.00005 and $\sin ^{2} \theta_{W}^{\text {eff lepton }}$ by -0.00020 . Let us comment on this evolution:

- The change in $\alpha\left(M_{Z}^{2}\right)$ is due to improvements in the theoretical predictions and experimental low-energy measurements over last 25 years, and following update in the used parametrization from [75] to [73].

- The $\Delta r$ and $\Delta r_{\text {rem }}$, which are displayed separately, represent gauge invariant corrections to $M_{W}$ calculation as shown in formulas (6) and (8). The $\Delta r$ is affected by options used for calculating $\Delta r_{L}$, which depends on the flag AMT4 used. It also depends on the parametrization of $\Delta \alpha\left(M_{Z}\right)$. The sensitivity of the $\Delta r_{r e m}$ to all changed introduced between $v 6.21$ and $v 6.45$ is almost negligible.

- As a consequence of different predicted $M_{W}$, the on-shell $s_{W}^{2}$ has evolved as well.

- Evolution of $\sin ^{2} \theta_{W}^{e f f} f$, illustrated in Fig. 2, comes from changing $s_{W}^{2}$ and $\mathscr{K}^{f}(s, t)$ form factors. It impacts $P^{\tau}, A_{F B}, \sigma^{\text {tot }}$ too.

In Table 8, we document impact of changing only parametrization of $\Delta \alpha_{h a d}^{(5)}(s)$, with other parameters and flags unchanged. Dominant effect comes from EW corrections to $M_{W}$, which shifts its value by $+8.4 \mathrm{MeV}$, reflected in change of $s_{W}^{2}$ by -0.00016 . The impact on the form factors is less significant and final shift in the $\sin ^{2} \theta_{W}^{\text {efflepton }}\left(M_{Z}^{2}\right)$ is of -0.00023 .

In Table 9, we document impact of changing only two-loop corrections to $M_{W}$, with other parameters and flags unchanged. The resulting shift on $M_{W}$ is smaller, $-2.9 \mathrm{MeV}$ only, resulting in +0.00006 shift on $s_{W}^{2}$ and, while multiplied with form factors which also have changed, correspondingly in -0.00008 shift on $\sin ^{2} \theta_{W}^{\text {eff lepton }}\left(M_{Z}^{2}\right)$.

B.3 Parametric uncertainties on $\sin ^{2} \theta_{W}^{e f f}\left(M_{Z}^{2}\right)$ predictions

We have studied dominant parametric uncertainties from $\Delta \alpha_{h}^{(5)}\left(M_{Z}^{2}\right)$ and $m_{t}$ for $\sin ^{2} \theta_{W}^{e f f}\left(M_{Z}^{2}\right)$ prediction. Recent detailed discussion on the parametric uncertainties of SM parameters can be found in [4]. Both components of $\sin ^{2} \theta_{W}^{e f f}\left(M_{Z}^{2}\right)=\operatorname{Re} \mathscr{K}^{f}\left(M_{Z}^{2}, \frac{-M_{Z}^{2}}{2}\right) \cdot s_{W}^{2}$ definition are sensitive to parametric uncertainties. 
Table 8 The DIZET 6.45 predictions for two different parametrizations of $\Delta \alpha_{h}^{(5)}\left(M_{Z}^{2}\right)$

\begin{tabular}{lccc}
\hline Parameter & $\begin{array}{c}\Delta \alpha_{h}^{(5)}\left(M_{Z}^{2}\right)=0.028040 \\
\text { (param. Jegerl. 1995) }\end{array}$ & $\begin{array}{l}\Delta \alpha_{h}^{(5)}\left(M_{Z}^{2}\right)=0.027576 \\
\text { (param. Jegerl. 2017) }\end{array}$ & $\Delta$ \\
\hline$\alpha\left(M_{Z}^{2}\right)$ & 0.0077587482 & 0.0077549256 & \\
$1 / \alpha\left(M_{Z}^{2}\right)$ & 128.88676996 & 128.95030224 & $+8.4 \mathrm{MeV}$ \\
$M_{W}(\mathrm{GeV})$ & 80.350538 & 80.358936 & -0.00016 \\
$\Delta r$ & 0.03690873 & 0.03640338 & -0.00016 \\
$\Delta r_{\text {rem }}$ & 0.01168001 & 0.01167960 & -0.00016 \\
$s_{W}^{2}$ & 0.22356339 & 0.22340108 & -0.00016 \\
$\sin ^{2} \theta_{W}^{\text {eff lepton }}\left(M_{Z}^{2}\right)$ & 0.23166087 & 0.23149900 & 0.23139248 \\
$\sin ^{2} \theta_{W}^{\text {eff up-quark }}\left(M_{Z}^{2}\right)$ & 0.23155425 & 0.23126543 & \\
$\sin ^{2} \theta_{W}^{\text {eff down-quark }}\left(M_{Z}^{2}\right)$ & 0.23142705 & & \\
\hline
\end{tabular}

Other flags as in Table 5

Table 9 The DIZET 6.45 predictions with improved treatment of two-loop corrections

\begin{tabular}{lccc}
\hline Parameter & AMT4 $=4$ & AMT4 $=8$ & $\Delta$ \\
\hline$\alpha\left(M_{Z}^{2}\right)$ & 0.0077549256 & 0.0077549256 & \\
$1 / \alpha\left(M_{Z}^{2}\right)$ & 128.95030206 & 128.95030224 & $-2.9 \mathrm{MeV}$ \\
$M_{W}(\mathrm{GeV})$ & 80.361846 & 80.358936 & 0.03640338 \\
$\Delta r$ & 0.03640338 & 0.01167960 & +0.00006 \\
$\Delta r_{\text {rem }}$ & 0.01167960 & 0.22340108 & -0.00008 \\
$s_{W}^{2}$ & 0.22333971 & 0.23149900 & -0.00008 \\
$\sin ^{2} \theta_{W}^{\text {eff lepton }}\left(M_{Z}^{2}\right)$ & 0.23157938 & 0.23139248 & -0.00008 \\
$\sin ^{2} \theta_{W}^{\text {eff } \text { up-quark }}\left(M_{Z}^{2}\right)$ & 0.23147290 & 0.23126543 & \\
$\sin ^{2} \theta_{W}^{\text {eff down-quark }}\left(M_{Z}^{2}\right)$ & 0.23134590 & & \\
\hline
\end{tabular}

Other flags as in Table 5

- In Table 10, we show impact of changing $\Delta \alpha_{h}^{(5)}\left(M_{Z}^{2}\right) \pm 0.0001$, which is the uncertainty of the parametrization of [73]. The resulting uncertainty on $\sin ^{2} \theta_{W}^{\text {eff }}\left(M_{Z}^{2}\right)$ is of \pm 0.000035

- In Table 11, we show impact of changing $m_{t} \pm 0.5 \mathrm{GeV}$, which is roughly the anticipated uncertainty of the measurements at LHC [92]. The resulting uncertainty on $\sin ^{2} \theta_{W}^{\text {eff }}\left(M_{Z}^{2}\right)$ is of \pm 0.000016 .

The total parametric uncertainty, added in quadrature, on $\sin ^{2} \theta_{W}^{e f f}\left(M_{Z}^{2}\right)$ is about \pm 0.00005 .

\section{Technical documentation of upgrades for TAUSPINNER electroweak re-weighting code}

Re-weighting of previously generated events is mathematically well defined procedure, whenever it can be assured that the weights (as in documenting TauSpinner Refs. [21,22]) are 
Table 10 The DIZET 6.45 predictions: shifts of selected parameters with variation of $\Delta \alpha_{h}^{(5)}\left(M_{Z}^{2}\right)$ from 0.0275762 (Ref. [73]) by \pm 0.0001

\begin{tabular}{lcccc}
\hline Parameter & $\Delta \alpha_{h}^{(5)}\left(M_{Z}^{2}\right)-0.0001$ & $\Delta \alpha_{h}^{(5)}\left(M_{Z}^{2}\right)=0.0275762$ & $\Delta \alpha_{h}^{(5)}\left(M_{Z}^{2}\right)+0.0001 \Delta / 2$ \\
\hline$\alpha\left(M_{Z}^{2}\right)$ & 0.0077541016 & 0.0077549256 & 0.0077557498 & \\
$1 / \alpha\left(M_{Z}^{2}\right)$ & 128.96400565 & 128.95030224 & 128.93659846 & $1.8 \mathrm{MeV}$ \\
$M_{W}(\mathrm{GeV})$ & 80.360747 & 80.358936 & 80.357124 & 0.03651261 \\
$\Delta r$ & 0.03629414 & 0.03640338 & 0.01167938 & 0.000035 \\
$\Delta r_{\text {rem }}$ & 0.01167983 & 0.01167960 & 0.22343610 & 0.000035 \\
$s_{W}^{2}$ & 0.22336607 & 0.22340108 & 0.23153392 & 0.000035 \\
$\sin ^{2} \theta_{W}^{\text {eff lepton }}\left(M_{Z}^{2}\right)$ & 0.23146409 & 0.23149900 & 0.23142737 & 0.000035 \\
$\sin ^{2} \theta_{W}^{\text {eff up-quark }}\left(M_{Z}^{2}\right)$ & 0.23135758 & 0.23139248 & 0.23130029 & \\
$\sin ^{2} \theta_{W}^{\text {eff down-quark }}\left(M_{Z}^{2}\right)$ & 0.23123057 & 0.23126543 & & \\
\hline
\end{tabular}

Table 11 The DIZET 6.45 predictions: shifts of selected parameters with variation of top-quark mass from $m_{t}=173.0 \mathrm{GeV}$ by $\pm 0.5 \mathrm{GeV}$

\begin{tabular}{lcccc}
\hline Parameter & $m_{t}-0.5 \mathrm{GeV}$ & $m_{t}=173.0 \mathrm{GeV}$ & $m_{t}+0.5 \mathrm{GeV}$ & $\Delta / 2$ \\
\hline$\alpha\left(M_{Z}^{2}\right)$ & 0.0077549221 & 0.0077549256 & 0.0077549291 & \\
$1 / \alpha\left(M_{Z}^{2}\right)$ & 128.95036003 & 128.95030224 & 128.95024461 & \\
$M_{W}(\mathrm{GeV})$ & 80.355935 & 80.358936 & 80.361941 & $3 \mathrm{MeV}$ \\
$\Delta r$ & 0.03658500 & 0.03640338 & 0.03622132 & \\
$\Delta r_{r e m}$ & 0.01167011 & 0.01167960 & 0.01168907 & 0.000058 \\
$s_{W}^{2}$ & 0.22345908 & 0.22340108 & 0.22334300 & 0.000016 \\
$\sin ^{2} \theta_{W}^{\text {eff lepton }}\left(M_{Z}^{2}\right)$ & 0.23151389 & 0.23149900 & 0.23148410 & 0.000016 \\
$\sin ^{2} \theta_{W}^{\text {eff } \text { up-quark }}\left(M_{Z}^{2}\right)$ & 0.23140736 & 0.23139248 & 0.23137758 & 0.000016 \\
$\sin ^{2} \theta_{W}^{\text {eff down-quark }}\left(M_{Z}^{2}\right)$ & 0.23128031 & 0.23126543 & 0.23125053 & \\
\hline
\end{tabular}

the ratios of matrix elements squared. Sums over spin degrees of freedom can be performed but no partial integration. If partial integration was performed, then difficulty to control biases would appear.

In Tauspinner, the Improved Born of (1) is coded as default for its nonSMBorn function. If form factors are available, look-up tables present, then they will be used for re-weighting algorithm. At default, it will be then assumed ${ }^{7}$ that sample was generated with Effective Born Tauola/LEP variant, see Table 1. A wealth of options is available for EW form-factor calculation, see Sect. B.1, Table 5 and Sect. F. Choice of options simplifying Improved Born to the cases closer, or to Effective Born itself, is listed in Table 12.

To monitor in a quick manner look-up tables with form factors, root scripts FFdraw . C and FFdrawDwa. C are provided, see Sect. 5. These scripts provide semi-analytical results for Born-level cross section, $A_{F B}$ and $A_{p o l}$ as a function of centre-of-mass energy, for incoming $e^{+} e^{-}$(or $\left.u \bar{u}, d \bar{d}\right)$ pairs. Two versions can be compared, e.g. EW improved Born with default Effective Tauola / LEP Born of Tauspinner, or of two variants of EW initialization.

7 If it is not the case, the weight will not be appropriate if TauSpinner initialization is not adjusted. 
Table 12 Initialization variants for non-standard Born of quark-level Drell-Yan $2 \rightarrow 2$ processes

\begin{tabular}{llll}
\hline KEYGSW & A: VVCor & B: propagator & C: form factors \\
\hline 0 & 1 & $\begin{array}{c}\text { Photon propagator } \\
\text { off, fixed } \Gamma_{Z} \\
\text { Running } \Gamma_{Z},\end{array}$ & All FFi=1 \\
1 & on & Fixed FFi from EW tables \\
2 & 1 & Running $\Gamma_{Z}$ & All FFi=1 \\
3 & 1 & All FFi=1 but FF $6=\Pi_{\gamma \gamma}\left(M_{Z}^{2}\right)$ \\
4 & 1 & Running $\Gamma_{Z}$ & All FFi from EW tables calculated at $\left(M_{Z}^{2},-M_{Z}^{2} / 2\right)$ \\
5 & 1 & Fixed $\Gamma_{Z}$, re-scaled & All FFi=1 \\
10 & 1 & Running $\Gamma_{Z}$ & All FFi=1, can be used for Effective Born $v 0$ \\
11 & 1 & Running $\Gamma_{Z}$ & FFi set as for Effective Born $v 1$ \\
12 & 1 & Running $\Gamma_{Z}$ & FFi set as for Effective Born $v 2$ \\
13 & 1 & with $\left.1 M_{Z}^{2}\right)$
\end{tabular}

It can be used to impose with the event weight EW loop effects on event samples. Variants are steered by the keyGSW parameter. Corresponding code is stored in: (A) - INITWKSDELT, (B) - T_BORNEW and (C) EWtables. Cxx. Fixed, running and fixed re-scaled $\Gamma_{Z}$ correspond, respectively, to Eqs. (11), (12) and (13). Further combination of options can be set by the simple re-coding. The initialization of effective Tauola Born is not affected by these options. It is performed elsewhere. For KEYGSW=11, 12, 13, when mode=0 results of Effective Born variants v0, v1, v2 can be obtained

In the distribution tar-ball [94] of Tauola/TauSpinner there are two example main programs, which demonstrate how re-weighting of EW effects is implemented (on the LHE and HepMC event formats) and how analytically these effects can be monitored. These programs, respectively, tau-reweight-test.cxx and Dizet-example/table-parsing-test. cxx need explanation because they evolved with time and more initialization options were introduced. In particular, to evaluate numerical differences between Effective Born and EW Improved Born as well as of the intermediate variants, the steering flag for the variants is keyGSW. Note that to re-weight, program needs to flip for each event, initialization between two variants and save some intermediate results to avoid massive recalculations. The semi-analytical table-parsing-test.cxx is obviously much faster. It is useful for study of small effects and not only to check correctness for EW form-factor tabulation.

Let us collect technical details for these programs and dependencies between routines:

- Dizet-example/table-parsing-test.cxx. This semi-analytic program is fast, does not require continuous interchanging between variants of initialization and is suitable to study small variation for predictions due to initialization fine tuning. It can be set for incoming $e^{+} e^{-}$(or $u \bar{u}, d \bar{d}$ ) pairs. It is obviously not suited for phase space integration over regions of experimental selection cuts.

- In tau-reweight-test. cxx, event re-weighting is demonstrated (LHE or HepMC format can be used), and for each event, two variants of Born are calculated. That is why flag keyGSW cannot be set once; method calculateWeightFromParticlesH() is executed twice ${ }^{8}$ for each event and ratio of the resulting weights is used.

At the beginning EWanomInit (defined locally in demo) is called and defines initial-

\footnotetext{
8 The getWtNonSM() returns weight for correction with respect to calculation of default SM calculation as initialized in tauola universal interface. It may be also suitable to activate call on EWreInit (1/2), for both variants of calculateWeightFromParticlesH() calculation. The EWreInit (1/2) is expected to be adjusted by the user to actual needs.
} 
Table 13 EW form factors of formula (1) calculated by DIZET and provided by KKMC tabulation code

\begin{tabular}{llll}
\hline Form factor & in KKMC in KKMC & in Dizet in example & in Effective Born \\
\hline$\rho_{\ell f}(s, t)$ & GSW (1) & FF1 & 1 \\
v1 & & & 1.005 \\
v2 & & & 1.005403 (up) \\
& & FF2 & 1.005889 (down) \\
$K_{e}(s, t)$ & GSW (2) & FF3 & 1 \\
$K_{f}(s, t)$ & GSW (3) & FF4 & 1 \\
$K_{e f}(s, t)$ & GSW (4) & - & 1 \\
- & - & FF6 & - \\
$\Pi_{\gamma \gamma}(s)$ & GSW (6) & & 1 \\
\hline
\end{tabular}

Names as used in: formula (1), KKMC tabulation code and in Dizet-example are collected. In Effective Born Eq. (5) numerical constants replace form factors of Improved Born (1). Depending on Effective Born version (see Table 1) $\sin ^{2} \theta_{W}$ of Improved Born is replaced by $\sin ^{2} \theta_{W}^{\text {eff }}$ or by flavour-dependent variants $\sin ^{2} \theta_{W}^{e f f l / u p / d o w}$, the $\rho_{\ell f}(s, t)$ is then replaced with constant $\rho_{\ell f}$. Similarly $\alpha(0)$ is replaced with $\alpha\left(M_{Z}\right)$

ization variant with the help of ExtraEWparamsSet ( ) method.

Next, for each event in the loop, EWreInit (it is expected to be adjusted by the user) re-initializes keyGSW and other parameters, with the, otherwise dummy, call on sigbornsdelt.

- One should note that sigbornsdelt call in tau-reweight-test.cxx returns dummy variable. The call is to pass keyGSW only. Returned value is used in Dizet-example/table-parsing-test.cxx though.

- In all the code of src/ew_born.cxx and src/tau_reweight_lib.cxx the keyGSW is not used. Corresponding routines are EW variants independent.

The src / ini twsw . $f$ is the file where routines for calculating Effective and/or Improved Born are placed. In the src/nonSM.cxx file, the method default_nonSM_bornz ( ) resides. The class data member variable m_keyGSW is used for keyGSW, and it is accessed with the help of the keyGSWGet (keyGSW) method; options are collected in Table 12. In Table 13, names of variables corresponding to the form factors, introduced in Eq. (1) of Improved Born, which are also used in KKMC and in Dizet-example scripts of TauSpinner, are explained. These form factors are used when t_bornew_calculation is invoked.

From the point of view of EW calculations, most of the variants are set and stored in EWtables.cxx. In particular, class/file variable m_keyGSW is stored among other variables of initialization. To access or modify, ExtraEWparamsGet and ExtraEWparams Set are prepared. These parameters used in initEWff passed with ExtraEWparamsGet in particular keyGSW are passed to initwkswdelt_

In the EWtables.cxx, the code for sigbornswdelt and AsNbornswdelt is stored. Through these methods keyGSW is passed to t_bornew_. The AsNbornswdelt, a near clone of sigbornswdelt, is used in drawing scripts for asymmetries.

It is important to note that the functions t_born and $t \_b o r n n e w$ which are used in tauola universal interface [95] and in Tauspinner are normalized to lowest order Born cross section, of photon exchange only and mass effects excluded. That is, $\frac{d \sigma_{\text {Born }}^{q \bar{q}}}{d \cos \theta}(s, \cos \theta, p)$ multiplied by $\frac{2 s}{\pi \alpha^{2}}\left(\alpha=\alpha_{Q E D}(0)\right.$. Thus, Born expression used in the 
weight calculations should approach $q_{f}^{2} q_{l}^{2}\left(1+\cos ^{2} \theta\right)$ at low energies. This condition, defines normalization for the EW-improved (or other, e.g. non-SM variant) of user provided Born. ${ }^{9}$ This is important if weights are used to relate cross sections obtained from $t$ _born and $t \_$bornnew. It is of no importance if only spin weight or weight based solely on user provided Born is used. This reference t_born of Effective Born (inherited from Tauola) can be easily modified/replaced by the user, as well as is the case with independently initialized t_bornnew of Improved Born.

In TauSpinner option to improve precision of generated MC events and re-weight from "fixed" to "running" width propagator, see Appendix E for explanation, is available. For that TauSpinner initialization with KEYGSW=11, KEYGSW=10 and KEYGSW=2 is prepared. For KEYGSW=11, KEYGSW=12 and KEYGSW=13 results corresponding to Effective Born, variants $v 0, v 1, v 2$, respectively, can be obtained, see Table 12. Let us explain now meaning of all other sigbornswdelt(mode, ID, S, CC, SWeff, DeltSQ, DeltV, Gmu, alfinv, AMZ00, GAM00, KEYGSW) input parameters. mode can be set to 0 or 1 . In the second case SWeff, AMZ00, GAM00 for $\sin ^{2} \theta_{W}^{e f f}, M_{Z}, \Gamma_{Z}$ will be overwritten with values stored in EW tables calculated with the DIZET library. ID $=0$, 1,2 denotes that calculation is performed, respectively, for outgoing lepton or down/up quark pair. The $\mathrm{S}, \mathrm{CC}$ denote Mandelstam variable and scattering angle. Anomalous coupling $\delta_{S 2 W}$ and $\delta_{V}$ of [22] Appendix B, are initialized with DeltSQ, Del tV, respectively, finally also $G_{F}, 1 / \alpha$ with $\mathrm{Gmu}$, alfinv.

The AsNbornswdelt ( ) features the same set of input parameters, but returns difference for cross section of forward and backward hemispheres instead of the sum.

For important technical details, README files and comments in the code of the distribution tar-ball [94] can be helpful.

\section{Initialization of variants for EW Improved Born}

In the previous Appendix we have completed presentation of some easy to activate in TauSpinner options. In many cases, it is sufficient to change some well-defined keys and/or input parameters such as $Z$ boson mass or some couplings. This of course shifts the results. The $\sigma^{t o t}, A_{F B}$ and $P_{\tau}$ predictions at the $Z$-pole are collected in Table 14, for incoming $e^{+} e^{-}$, up or down quarks.

Some options, e.g. KEYGSW=0, 2, 4, 10, are prepared for technical tests, rather than for evaluation of physics uncertainties, because then parts of matrix elements are used only. Other options, when matrix elements truncated to some level of approximations, are then more useful. All these options are useful for test of particular parts of EW predictions obtained with a given version of EW form factors. This supplements discussed earlier options of EW form-factor initialization, see Appendix B.1 and Table 5. Further, of more historical nature tests, with EW form factors calculated with older versions of EW DIZET library presented in Appendix F, are collected in Fig. 8 and Table 4. Results of the present Appendix are to supplement discussion of reliability and limitation of the Effective Born variants as compared with Improved Born, in general and in the context of particular applications.

The Effective Born, variants $\mathrm{v} 0, \mathrm{v} 1, \mathrm{v} 2$, require change of input parameters. Then, mode $=0$ and KEYGSW at 11,12 or 13 should be, respectively, set. One should notice some shifts of Table 14 results with respect to the ones presented in Tables 2 and 3. Note that we do not average over energy ranges and incoming quark flavours now.

9 The method for non-SM Born can be replaced with the pointer to the one of user choice. 
Table 14 Numerical results for initialization variants as explained in Table 12

\begin{tabular}{|c|c|c|c|c|c|c|}
\hline KEYGSW & & $\frac{\sigma_{\text {tot }}^{e^{+} e^{-}}\left(M_{Z}\right)}{\sigma_{\text {tot improved }}^{e^{+} e^{-}}\left(M_{Z}\right)}$ & & $P_{\tau}^{e^{+} e^{-}}\left(M_{Z}\right)$ & & $A_{F B}^{e^{+} e^{-}}\left(M_{Z}\right)$ \\
\hline 0 & & 0.989939 & & 0.1463264 & & 0.0161546 \\
\hline 1 & & 1.000000 & & 0.1449616 & & 0.0177039 \\
\hline 2 & & 1.000736 & & 0.2093134 & & 0.0330312 \\
\hline 3 & & 1.001438 & & 0.2094436 & & 0.0349617 \\
\hline 4 & & 1.011539 & & 0.2093809 & & 0.0344227 \\
\hline 5 & & 1.000001 & & 0.1449558 & & 0.0176505 \\
\hline 10 & & 1.002227 & & 0.2093154 & & 0.0330336 \\
\hline 11 & & 1.000736 & & 0.2093134 & & 0.0330312 \\
\hline $11 \mathrm{v} 0$ & & 0.9899340 & & 0.1463264 & & 0.0161546 \\
\hline $12 \mathrm{v} 1$ & & 0.9999098 & & 0.1463351 & & 0.0161556 \\
\hline $13 \mathrm{v} 2$ & & 0.9999098 & & 0.1463351 & & 0.0161556 \\
\hline KEYGSW & $\frac{\sigma_{\text {tot }}^{u \bar{u}}\left(M_{Z}\right)}{\sigma_{\text {tot impr. }}^{u \bar{u}}\left(M_{Z}\right)}$ & $P_{\tau}^{u \bar{u}}\left(M_{Z}\right)$ & $A_{F B}^{u \bar{u}}\left(M_{Z}\right)$ & $\frac{\sigma_{\text {tot }}^{d \bar{d}}\left(M_{Z}\right)}{\sigma_{\text {tot impr. }}^{d \bar{d}}\left(M_{Z}\right)}$ & $P_{\tau}^{d \bar{d}}\left(M_{Z}\right)$ & $A_{F B}^{d \bar{d}}\left(M_{Z}\right)$ \\
\hline 0 & 0.989244 & 0.146859 & 0.073530 & 0.988081 & 0.1471353 & 0.1032379 \\
\hline 1 & 1.000000 & 0.146990 & 0.074664 & 1.000000 & 0.1476778 & 0.1038546 \\
\hline 2 & 1.009430 & 0.209984 & 0.109447 & 1.003882 & 0.2103266 & 0.1483706 \\
\hline 3 & 1.009759 & 0.210529 & 0.110548 & 1.003976 & 0.2107218 & 0.1488000 \\
\hline 4 & 1.020676 & 0.210387 & 0.110274 & 1.015833 & 0.2106413 & 0.1487130 \\
\hline 5 & 0.999999 & 0.146974 & 0.074633 & 0.999999 & 0.1476667 & 0.1038425 \\
\hline 10 & 1.010939 & 0.209986 & 0.109449 & 1.005384 & 0.2103272 & 0.1483712 \\
\hline 11 & 1.009430 & 0.209985 & 0.109447 & 1.003882 & 0.2103266 & 0.1483706 \\
\hline $11 \mathrm{v} 0$ & 0.989244 & 0.146859 & 0.073530 & 0.988081 & 0.1471353 & 0.1032379 \\
\hline $12 \mathrm{v} 1$ & 0.999244 & 0.146863 & 0.073532 & 0.998087 & 0.1471360 & 0.1032383 \\
\hline $13 \mathrm{v} 2$ & 1.000127 & 0.146863 & 0.073573 & 1.000039 & 0.1471361 & 0.1032550 \\
\hline
\end{tabular}

Numerical results for $\mathrm{v} 0, \mathrm{v} 1, \mathrm{v} 2$ are also provided, then in addition to KEYGSW $=11,12$ or 13, input parameters for sigbornswdelt ( ), AsNbornswdelt ( ) need to be adjusted and mod $=0$. The $\alpha\left(M_{Z}^{2}\right) / \alpha(0)$ factors entering cross sections normalization are dropped out from the $\sigma^{\text {tot }}$ ratios

\section{E The $s$-dependent Z-boson width}

In formula (1) for the definition of $Z$ propagator running width is used:

$$
\chi_{Z}(s)=\frac{1}{s-M_{Z}^{2}+i \cdot \Gamma_{Z} \cdot s / M_{Z}} .
$$

The form factors of Eq. (1) are calculated for the on mass-shell (nominal) value of $M_{Z}$. The introduction of so-called $s$-dependent width is equivalent to partial re-summation to higher orders of dominant loop correction: the boson $s$-dependent self-energy. In fact such re-summation, running $Z$ width, was used in many analyses of LEP 1 era. 
However, in Monte Carlos and strong interaction calculations of LHC era, the $Z$ propagator of constant width is often used:

$$
\chi_{Z}^{\prime}(s)=\frac{1}{s-M_{Z}^{2}+i \cdot \Gamma_{Z} \cdot M_{Z}} .
$$

One can ask the question, how analytic forms of (11) and (12) translate to each other. In fact, this translation is well known at least since Ref. [96] published more than 30 years ago, but let us readdress it for the reference again. From Eq. (11), we obtain Eq. (12) if the following re-definitions are used

$$
\begin{aligned}
\chi_{Z}(s) & =\frac{1}{s\left(1+i \cdot \Gamma_{Z} / M_{Z}\right)-M_{Z}^{2}} \\
& =\frac{\left(1-i \cdot \Gamma_{Z} / M_{Z}\right)}{s\left(1+\Gamma_{Z}^{2} / M_{Z}^{2}\right)-M_{Z}^{2}\left(1-i \cdot \Gamma_{Z} / M_{Z}\right)} \\
& =\frac{\left(1-i \cdot \Gamma_{Z} / M_{Z}\right)}{\left(1+\Gamma_{Z}^{2} / M_{Z}^{2}\right)} \frac{1}{s-\frac{M_{Z}^{2}}{1+\Gamma_{Z}^{2} / M_{Z}^{2}}+i \cdot \frac{\Gamma_{Z} M_{Z}}{1+\Gamma_{Z}^{2} / M_{Z}^{2}}} \\
& =N_{Z}^{\prime} \frac{1}{s-M_{Z}^{\prime 2}+i \Gamma_{Z}^{\prime} M_{Z}^{\prime}} \\
M_{Z}^{\prime} & =\frac{M_{Z}}{\sqrt{1+\Gamma_{Z}^{2} / M_{Z}^{2}}} \\
\Gamma_{Z}^{\prime} & =\frac{\Gamma_{Z}}{\sqrt{1+\Gamma_{Z}^{2} / M_{Z}^{2}}} \\
N_{Z}^{\prime} & =\frac{\left(1-i \cdot \Gamma_{Z} / M_{Z}\right)}{\left(1+\Gamma_{Z}^{2} / M_{Z}^{2}\right)}=\frac{\left(1-i \cdot \Gamma_{Z}^{\prime} / M_{Z}^{\prime}\right)}{\left(1+\Gamma_{Z}^{\prime 2} / M_{Z}^{\prime 2}\right)}
\end{aligned}
$$

The $s$-dependent width in $Z$ propagator translates into mass and width shift and introduction of the complex factor in front of the constant width $Z$ propagator. This last point is possibly least trivial as it effectively means re-definition of $Z$ coupling. That is why it cannot be understood as parameter re-scaling. It points to presence in higher-order relations between vacuum polarization and vertex. Most of the changes are due to the term $\Gamma_{Z}^{2} / M_{Z}^{2}$ except of the overall phase which result from $1-i \cdot \Gamma_{Z} / M_{Z}$ factor and which change the $\gamma-Z$ interference. The shift in $M_{Z}$ is by about $34 \mathrm{MeV}$ downwards, and the shift in $\Gamma_{Z}$ by $1 \mathrm{MeV}$, due to the re-parametrization of the Z-boson propagator.

In Fig. 9, comparison of the cross sections and $A_{f b}$ for DY production in $p p$ collisions for different implementations of $\chi_{Z}(s)$ is shown. Dashed line of reference corresponds to using formula (11). Green line to complete formula (13). Red line corresponds to formula (13) but without $N_{Z}^{\prime}$ scaling and blue line to formula (12), with nominal $M_{Z}$ and $\Gamma_{Z}$.

It is common for LHC MC generators to use formula (12) for $Z$ propagator, with $M_{Z}$ and $\Gamma_{Z}$ of nominal, on-mass-shell values. Numerically this is better approximation than with shifted $M_{Z}$ and $\Gamma_{Z}$ but $N_{Z}^{\prime}$ missing. This observation is true both for EW LO and EW corrected calculations; for cross section and $A_{F B}$. Quantitative estimates are collected in Tables 15 and 16 .

Note that when options of "running $\Gamma_{Z}$ " and " fixed $\Gamma_{Z}$ " are compared, the same EW corrections in both cases tuned to "running $\Gamma_{Z}$ " convention are used. It is beyond the scope of the paper to investigate, how $\mathrm{NLO}+\mathrm{HO}$ corrections, calculated with the fixed width/ pole 
Fig. 9 Ratio of the cross sections (top) and $\Delta A_{f b}$ (bottom) for $\mathrm{EW}$ LO but different forms of Z-boson propagator, see text. The $\mathrm{MC} p p \rightarrow Z j ; Z \rightarrow l^{+} l^{-}$ events were used for estimations
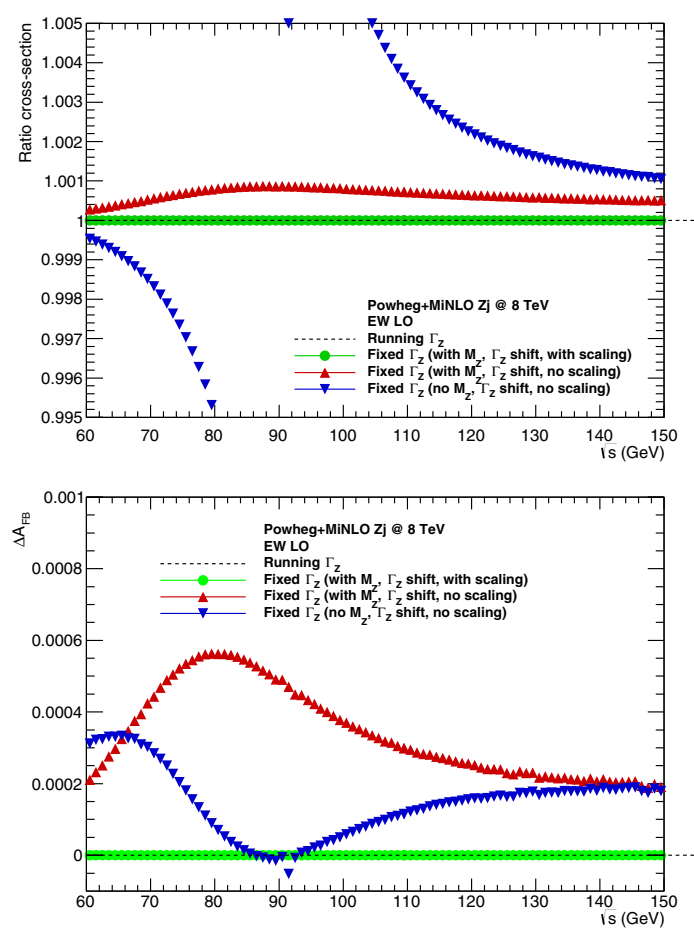

Table 15 Ratio of the cross sections $(\sigma)$, calculated with different forms of $Z$-boson propagator and integrated over outgoing lepton pair mass windows

\begin{tabular}{lccccc}
\hline$\sigma($ Fixed $) / \sigma$ (Running) $m_{e e}$ ranges (in GeV) & $90.5-91.5$ & $89-93$ & $60-81$ & $81-101$ & $101-150$ \\
At EW LO & & & & & \\
With $M_{Z}, \Gamma_{Z}$ shift, no $N_{Z}^{\prime}$ & 1.00087 & 1.00087 & 1.00062 & 1.00086 & 1.00071 \\
No $M_{Z}, \Gamma_{Z}$ shift, no $N_{Z}^{\prime}$ & 0.99620 & 1.00074 & 0.99716 & 0.99977 & 1.00392 \\
At EW NLO + HO & & & & & 1.00003 \\
With $M_{Z}, \Gamma_{Z}$ shift, no $N_{Z}^{\prime}$ & 1.00113 & 1.00085 & 1.00043 & 1.0003 \\
No $M_{Z}, \Gamma_{Z}$ shift, no $N_{Z}^{\prime}$ & 0.99746 & 1.00122 & 0.99719 & 1.00013 & 1.00392 \\
\hline
\end{tabular}

It is shown for EW LO and EW NLO+HO predictions of $O(\alpha(0) \mathrm{EW}$ scheme for $p p$ collisions at $8 \mathrm{TeV}$ centre of mass energy, while EW NLO $+\mathrm{HO}$ corrections are tuned to running $\Gamma_{Z}$ convention

mass convention, gradually mitigate (as they should) discrepancy observed at EW LO level between Eqs. (11) and (13) definition of $Z$ propagator without $N_{Z}^{\prime}$ included.

\section{F Versions of DIZET library}

In the KKMC distribution tar-ball, explained in Ref. [97], the code for calculation of EW corrections is stored in directory $\mathrm{KK}-\mathrm{all} / \mathrm{dizet}$. The program stored in that directory calculates EW form factors and writes them into ASCII format text files. To change the version of the form factors requires simply use of these tables calculated with different 
Table 16 Difference of $A_{f b}$ calculated for different forms of $Z$-boson propagator and for integrated outgoing lepton pair mass windows

\begin{tabular}{lllllr}
\hline $\begin{array}{l}A_{f b} \text { (Running) }-A_{f b} \text { (Fixed) } \\
m_{e e} \text { ranges (in GeV) }\end{array}$ & $90.5-91.5$ & $89-93$ & $60-81$ & $81-101$ & $101-150$ \\
At EW LO & & & & & \\
With $M_{Z}, \Gamma_{Z}$ shift, no $N_{Z}^{\prime}$ & -0.00048 & -0.00047 & -0.00047 & -0.00047 & -0.00030 \\
No $M_{Z}, \Gamma_{Z}$ shift, no $N_{Z}^{\prime}$ & -0.00006 & -0.00026 & -0.00012 & -0.00040 & -0.00005 \\
At EW NLO+HO & & & & & \\
With $M_{Z}, \Gamma_{Z}$ shift, no $N_{Z}^{\prime}$ & -0.00053 & -0.00053 & -0.00052 & -0.00053 & -0.00024 \\
No $M_{Z}, \Gamma_{Z}$ shift, no $N_{Z}^{\prime}$ & -0.00007 & -0.00030 & -0.00026 & -0.00048 & -0.00004 \\
\hline
\end{tabular}

It is shown for EW LO and EW NLO+HO predictions of $O(\alpha(0) \mathrm{EW}$ scheme for $p p$ collisions at $8 \mathrm{TeV}$ centre-of-mass energy, while EW NLO $+\mathrm{HO}$ corrections are tuned to running $\Gamma_{Z}$ convention

versions of library or with different sets of initialization parameters. In parallel to program stored in KK-all/dizet which calculates EW form factors as stored with Ref. [98] the following new ones, di zet $-6.42-\mathrm{cpc}$, di zet-6.42 and dizet -6.45 , were prepared and can be used following old instruction of KKMC documentation. There was only one change introduced, convenient for table reading by the TauSpinner package [22], and the table write format for EW form factors was modified to KK-all/dizet/BornV.h and if tables are used with KKMC, the original one of KK-all/bornv/BornV. $h$ file should be re-installed. This is true for all mentioned above DIZET $6 . \mathrm{xx}$ variants.

- DIZET 6.21 (dizet) is distributed with KKMC through CPC EW corrections and in particular vacuum polarization was not updated for backup compatibility. This version of library is documented in [25]. Note that upgrades of EW corrections within LEP experiments were not always well documented. This is in particular true for the photon vacuum polarization $\Pi_{\gamma \gamma}(s)$.

- DIZET 6.42 (dizet-6.42-cpc) as in published ZFITTER [74]. This is the last published/archived version of DIZET code. Note that as a default $\Pi_{\gamma \gamma}$ from Ref. [75] is still mentioned but obviously it was upgraded for the final versions of LEP data analysis of Ref. [2].

- DIZET 6.42 (dizet-6.42) with $\Pi_{\gamma \gamma}$ updated to hadr5n17_compact.f of Ref. [99]. Parametrization taken from author web page, dated Oct 8 02:19:56 2017.

- DIZET 6.45 (dizet-6.45) VERSION 6.45 (30 August 2019) with the vacuum polarization code and fermionic two loops corrections, AMT4 flag upgraded by DIZET authors themselves.

Comparison of results for these versions is given in Fig. 8 and in Table 4.

\section{References}

1. ALEPH, CDF, D0, DELPHI, L3, OPAL, SLD, LEP Electroweak Working Group, Tevatron Electroweak Working Group, SLD Electroweak, Heavy Flavour Groups Collaboration. arXiv:1012.2367

2. SLD Electroweak Group, DELPHI, ALEPH, SLD, SLD Heavy Flavour Group, OPAL, LEP Electroweak Working Group, L3 Collaboration, S. Schael et al., Phys. Rept. 427, 257-454 (2006). arXiv:hep-ex/0509008

3. M. Davier, L. Duflot, F. Le Diberder, A. Rouge, Phys. Lett. B 306, 411-417 (1993) 
4. A. Blondel, J. Gluza, S. Jadach, P. Janot, T. Riemann, eds., Theory for the FCC-ee: Report on the 11th FCC-ee Workshop Theory and Experiments, vol. 3/2020 of CERN Yellow Reports: Monographs, Geneva, CERN, 5, 2019

5. [ATLAS], ATLAS-CONF-2018-037

6. A. M. Sirunyan et al. [CMS], Eur. Phys. J. C 78(9), 701 (2018). https://doi.org/10.1140/epjc/s10052018-6148-7 [arXiv:1806.00863 [hep-ex]]

7. [ATLAS], ATL-PHYS-PUB-2018-037

8. [CMS], CMS-PAS-FTR-17-001

9. DYu. Bardin, P. Christova, M. Jack, L. Kalinovskaya, A. Olchevski, S. Riemann, T. Riemann, Comput. Phys. Commun. 133, 229-395 (2001). arXiv:hep-ph/9908433

10. S. Jadach, B. Ward, Z. Was, Phys. Rev. D 63, 113009 (2001). arXiv:hep-ph/0006359

11. A. Akhundov, A. Arbuzov, S. Riemann, T. Riemann, Phys. Part. Nucl. 45(3), 529-549 (2014). https:// doi.org/10.1134/S1063779614030022. [arXiv:1302.1395 [hep-ph]]

12. See e.g. C. Itzykson, J.B. Zuber, "Quantum Field Theory,” Published in: New York, USA: Mcgraw-hill (1980) 705 P. (International Series In Pure and Applied Physics)

13. A. Denner, S. Dittmaier, Phys. Rep. 864, 1-163 (2020). https://doi.org/10.1016/j.physrep.2020.04.001. [arXiv:1912.06823 [hep-ph]]

14. A. Barroso et al., Electroweak radiative corrections at LEP energies, in ECFA Workshop: LEP 200, vol 4 (1987)

15. D.Y. Bardin, M. Grunewald, G. Passarino. arXiv:hep-ph/9902452

16. W. Hollik, U. Meier, S. Uccirati, Nucl. Phys. B 731, 213-224 (2005). arXiv:hep-ph/0507158

17. P. Gambino, A. Sirlin, Phys. Rev. D 49, 1160-1162 (1994). arXiv:hep-ph/9309326

18. E. Richter-Was, Z. Was, Eur. Phys. J. C 76(8), 473 (2016). arXiv: 1605.05450

19. E. Richter-Was, Z. Was, Eur. Phys. J. C 77(2), 111 (2017). arXiv:1609.02536

20. Z. Czyczula, T. Przedzinski, Z. Was, Eur. Phys. J. C 72, 1988 (2012). arXiv:1201.0117

21. T. Przedzinski, E. Richter-Was, Z. Was, Eur. Phys. J. C 79, 91 (2019). https://doi.org/10.1140/epjc/ s10052-018-6527-0. [arXiv:1802.05459 [hep-ph]]

22. E. Richter-Was, Z. Was, Eur. Phys. J. C 79, 480 (2019). arXiv:1808.08616

23. S. Jadach, B.F.L. Ward, Z. Wa̧s, Comput. Phys. Commun. 79, 503 (1994)

24. S. Jadach, Z. Wạs, B.F.L. Ward, Comput. Phys. Commun. 130, 260 (2000). Up to date source available from http://home.cern.ch/jadach/

25. DYu. Bardin, M.S. Bilenky, T. Riemann, M. Sachwitz, H. Vogt, Comput. Phys. Commun. 59, 303-312 (1990)

26. A. Arbuzov, S. Jadach, Z. Was, B.F.L. Ward, S.A. Yost, The Monte Carlo program KKMC, for the Lepton or quark pair production at LEP/SLC Energies-updated of electroweak calculations. arXiv:IFJPAN-IV-2020-05 BU-HEPP-2020-01

27. G. Passarino. arXiv:2104.13569 [hep-ph]

28. J.C. Collins, D.E. Soper, G.F. Sterman, Adv. Ser. Direct. High Energy Phys. 5, 1-91 (1989). https://doi. org/10.1142/9789814503266_0001. [arXiv:hep-ph/0409313 [hep-ph]]

29. L.N. Lipatov, Yad. Fiz. 20, 181-198 (1974)

30. Y.L. Dokshitzer, D. Diakonov, S.I. Troian, Phys. Rep. 58, 269-395 (1980). https://doi.org/10.1016/03701573(80)90043-5

31. G. Altarelli, Phys. Rep. 81, 1 (1982). https://doi.org/10.1016/0370-1573(82)90127-2

32. J. Collins, Camb. Monogr. Part. Phys. Nucl. Phys. Cosmol. 32, 1-624 (2011)

33. F.A. Berends, R. Kleiss, S. Jadach, Comput. Phys. Commun. 29, 185-200 (1983)

34. E. Mirkes, J. Ohnemus, Phys. Rev. D 51, 4891-4904 (1995). arXiv:hep-ph/9412289

35. J.C. Collins, D.E. Soper, Phys. Rev. D 16, 2219 (1977)

36. S. Banerjee, Z. Was, CERN Yellow Rep. Monogr. 3, 211-212 (2020)

37. S. Banerjee, J. Kalinowski, W. Kotlarski, T. Przedzinski, Z. Was, Eur. Phys. J. C 73, 2313 (2013). arXiv: 1212.2873

38. S. Alioli et al., Eur. Phys. J. C 77(5), 280 (2017). arXiv:1606.02330

39. S. Dittmaier, A. Huss, C. Schwinn, Nucl. Phys. B 885, 318-372 (2014). arXiv:1403.3216

40. S. Kallweit, J.M. Lindert, P. Maierhofer, S. Pozzorini, M. Schönherr, JHEP 04, 021 (2016). https://doi. org/10.1007/JHEP04(2016)021. [arXiv:1511.08692 [hep-ph]]

41. D. Y. Bardin, G. Passarino, "The standard model in the making: Precision study of the electroweak interactions," Published in: Oxford, UK: Clarendon (1999) 685 p, (International series of monographs on physics. 104)

42. G. Altarelli, Acta Phys. Austriaca Suppl. 24, 229-273 (1982). https://doi.org/10.1007/978-3-7091-40314_6

43. S. Bethke. https://doi.org/10.1142/9789813238053_0005. [arXiv:1712.03035 [hep-ex]] 
44. R.G. Stuart, Phys. Lett. B 272, 353-358 (1991). https://doi.org/10.1016/0370-2693(91)91842-J

45. B.A. Kniehl, R.G. Stuart, Comput. Phys. Commun. 72, 175-220 (1992). https://doi.org/10.1016/00104655(92)90149-S

46. A. Denner, J.N. Lang, Eur. Phys. J. C 75(8), 377 (2015). https://doi.org/10.1140/epjc/s10052-015-35792. [arXiv:1406.6280 [hep-ph]]

47. S. Schael et al. [ALEPH, DELPHI, L3, OPAL and LEP Electroweak]. Phys. Rep. 532, 119-244 (2013). https://doi.org/10.1016/j.physrep.2013.07.004. [arXiv:1302.3415 [hep-ex]]

48. M. Grazzini, S. Kallweit, J.M. Lindert, S. Pozzorini, M. Wiesemann, JHEP 02, 087 (2020). https://doi. org/10.1007/JHEP02(2020)087. [arXiv:1912.00068 [hep-ph]]

49. R. Stuart, Private communication, Munich (1988)

50. S. Jadach, B.F.L. Ward, Z. Was, Comput. Phys. Commun. 66, 276-292 (1991). https://doi.org/10.1016/ 0010-4655(91)90077-X

51. M. Greco, G. Pancheri-Srivastava, Y. Srivastava, Nucl. Phys. B 171, 118 (1980). [erratum: Nucl. Phys. B 197 (1982), 543]. https://doi.org/10.1016/0550-3213(82)90458-8

52. F.A. Berends, R. Kleiss, S. Jadach, Nucl. Phys. B 202, 63-88 (1982). https://doi.org/10.1016/05503213(82)90221-8

53. M. Bohm, W. Hollik, Nucl. Phys. B 204, 45-77 (1982). https://doi.org/10.1016/0550-3213(82)90421-7

54. R. Kleiss, W.J. Stirling, Nucl. Phys. B 262, 235 (1985)

55. S. Jadach, B.F.L. Ward, Z. Was, Eur. Phys. J. C 22, 423-430 (2001). https://doi.org/10.1007/ s100520100818. [arXiv:hep-ph/9905452 [hep-ph]]

56. D. Bardin, S. Jadach, T. Riemann, Z. Was, Eur. Phys. J. C 24, 373-383 (2002). https://doi.org/10.1007/ s100520200948. [arXiv:hep-ph/0110371 [hep-ph]]

57. Z. Was, Eur. Phys. J. C 44, 489-503 (2005). https://doi.org/10.1140/epjc/s2005-02381-y. [arXiv:hep-ph/0406045 [hep-ph]]

58. S. Jadach, Z. Was, Phys. Lett. B 219, 103-106 (1989). https://doi.org/10.1016/0370-2693(89)90847-2

59. S. Jadach, B. Pietrzyk, E. Tournefier, B.F.L. Ward, Z. Was, Phys. Lett. B 465, 254-259 (1999). https:// doi.org/10.1016/S0370-2693(99)01047-3. [arXiv:hep-ph/9907547 [hep-ph]]

60. S. Jadach, S. Yost, Phys. Rev. D 100(1), 013002 (2019). https://doi.org/10.1103/PhysRevD.100.013002. [arXiv: 1801.08611 [hep-ph]]

61. M.W. Grunewald, G. Passarino, E. Accomando, A. Ballestrero, P. Bambade, D.Y. Bardin, W. Beenakker, F.A. Berends, E. Boos, A. Chapovsky et al. arXiv:hep-ph/0005309 [hep-ph]

62. T.A. Aaltonen et al. [CDF and D0]. Phys. Rev. D 97(11) (2018). https://doi.org/10.1103/PhysRevD.97. 112007. [arXiv:1801.06283 [hep-ex]]

63. J. Alwall, M. Herquet, F. Maltoni, O. Mattelaer, T. Stelzer, JHEP 06, 128 (2011). https://doi.org/10.1007/ JHEP06(2011)128. [arXiv:1106.0522 [hep-ph]]

64. S. Frixione, P. Nason, C. Oleari, JHEP 11, 070 (2007). https://doi.org/10.1088/1126-6708/2007/11/070. [arXiv:0709.2092 [hep-ph]]

65. S. Alioli, P. Nason, C. Oleari, E. Re, JHEP 06, 043 (2010). https://doi.org/10.1007/JHEP06(2010)043. [arXiv:1002.2581 [hep-ph]]

66. D. Lombardi, M. Wiesemann, G. Zanderighi. arXiv:2010.10478 [hep-ph]

67. A. Denner, R. Franken, M. Pellen, T. Schmidt, JHEP 11, 110 (2020). https://doi.org/10.1007/ JHEP11(2020)110. [arXiv:2009.00411 [hep-ph]]

68. S. Dittmaier, Gauge-invariance issues in Drell-Yan production and solutions implemented in RADY, Talk LHC EWWG Meeting, Dec 18, 2020. https://indico.cern.ch/event/980547/contributions/4155193/ attachments/2165557/3654811/lhcewwg20.pdf

69. W. Beenakker, W. Hollik, Z. Phys. C 40, 141 (1988). https://doi.org/10.1007/BF01559728

70. DYu. Bardin, P.K. Khristova, O.M. Fedorenko, Nucl. Phys. B 175, 435-461 (1980)

71. DYu. Bardin, P.K. Khristova, O.M. Fedorenko, Nucl. Phys. B 197, 1-44 (1982)

72. A. Sirlin, Phys. Rev. D 22, 971-981 (1980)

73. F. Jegerlehner, EPJ Web Conf. 218, 01003 (2019). arXiv:1711.06089

74. A.B. Arbuzov, M. Awramik, M. Czakon, A. Freitas, M.W. Grunewald, K. Monig, S. Riemann, T. Riemann, Comput. Phys. Commun. 174, 728-758 (2006). arXiv:hep-ph/0507146

75. S. Eidelman, F. Jegerlehner, Z. Phys. C67, 585-602 (1995). arXiv:hep-ph/9502298

76. G. Degrassi, S. Fanchiotti, F. Feruglio, P. Gambino, A. Vicini, Phys. Lett. B 350, 75-84 (1995). arXiv:hep-ph/9412380

77. G. Degrassi, F. Feruglio, A. Vicini, S. Fanchiotti, P. Gambino, Two loop corrections for electroweak processes, in '95 Electroweak Interactions, Unified Theories. Proceedings, Leptonic Session of the 30th Rencontres de Moriond, Moriond Particle Physics Meetings, Les Arcs, France, March 11-18, 1995 (1995), pp. 77-85. arXiv:hep-ph/9507286

78. G. Degrassi, P. Gambino, A. Vicini, Phys. Lett. B 383, 219-226 (1996). arXiv:hep-ph/9603374 
79. G. Degrassi, P. Gambino, Nucl. Phys. B 567, 3-31 (2000). arXiv:hep-ph/9905472

80. A. Freitas, W. Hollik, W. Walter, G. Weiglein, Phys. Lett. B 495, 338-346 (2000). [Erratum: Phys. Lett. B 570, no.3-4, 265(2003)]. arXiv:hep-ph/0007091

81. A. Freitas, S. Heinemeyer, W. Hollik, W. Walter, G. Weiglein, Nucl. Phys. Proc. Suppl. 89, 82-87 (2000). arXiv:hep-ph/0007129

82. A. Freitas, W. Hollik, W. Walter, G. Weiglein, Nucl. Phys. B 632, 189-218 (2002). [Erratum: Nucl. Phys.B666,305(2003)]. arXiv:hep-ph/0202131

83. M. Awramik, M. Czakon, A. Freitas, G. Weiglein, Phys. Rev. D 69, 053006 (2004). arXiv:hep-ph/0311148

84. M. Awramik, M. Czakon, A. Freitas, G. Weiglein, Phys. Rev. Lett. 93, 201805 (2004). arXiv:hep-ph/0407317

85. M. Awramik, M. Czakon, A. Freitas, JHEP 11, 048 (2006). arXiv:hep-ph/0608099

86. I. Dubovyk, A. Freitas, J. Gluza, T. Riemann, J. Usovitsch, Phys. Lett. B 762, 184-189 (2016). arXiv: 1607.08375

87. I. Dubovyk, A. Freitas, J. Gluza, T. Riemann, J. Usovitsch, JHEP 08, 113 (2019). arXiv:1906.08815

88. L. Avdeev, J. Fleischer, S. Mikhailov, O. Tarasov, Phys. Lett. B 336, 560-566 (1994). [Erratum: Phys. Lett. B 349, 597 (1995)]. arXiv:hep-ph/9406363

89. P.A. Zyla et al. [Particle Data Group], PTEP 2020(8), 083C01 (2020). https://doi.org/10.1093/ptep/ ptaa104

90. ATLAS Collaboration, M. Aaboud et al. Phys. Lett. B 784, 345-366 (2018). arXiv: 1806.00242

91. ATLAS Collaboration, M. Aaboud et al., Eur. Phys. J. C78(2), 110 (2018). [Erratum: Eur. Phys. J. C78, no.11, 898 (2018)]. arXiv: 1701.07240

92. ATLAS Collaboration, M. Aaboud et al., Eur. Phys. J. C79(4), 290 (2019). arXiv:1810.01772

93. LHC EW WG. https://lpcc.web.cern.ch/electroweak-precision-measurements-lhc-wg(2018-2020)

94. N. Davidson, G. Nanava, T. Przedzinski, E. Richter-Was, Z. Was, TAUOLA and TAUOLA C++ Interface source code and documentation available from http://wasm.web.cern.ch/wasm/C++.html or http://tauolapp.web.cern.ch

95. N. Davidson, G. Nanava, T. Przedzinski, E. Richter-Was, Z. Was, Comput. Phys. Commun. 183, 821-843 (2012). arXiv: 1002.0543

96. D. Bardin, A. Leike, T. Riemann, M. Sachwitz, Phys. Lett. B 206, 539-542 (1988)

97. A. Arbuzov, S. Jadach, Z. Was, B. Ward, S. Yost, Comput. Phys. Commun. 260, 107734 (2021). arXiv:2007.07964

98. S. Jadach, B.F.L. Ward, Z. Was, Phys. Rev. D 88(11), 114022 (2013). arXiv:1307.4037

99. F. Jegerlehner, $\alpha_{Q E D, e f f}(\mathrm{~s})$ for precision physics at the FCC-ee/ILC, in Theory Report on the 11th FCC-ee Workshop (2019), pp. 9-37 